\title{
Competing first-price and second-price auctions
}

\author{
Joyce Delnoij ${ }^{1}(\mathbb{D}) \cdot$ Kris De Jaegher $^{2}$ (I)
}

Received: 15 February 2017 / Accepted: 29 October 2018 / Published online: 13 November 2018

(c) The Author(s) 2018

\begin{abstract}
This paper theoretically investigates which auctions are selected by competing sellers when they can choose between first-price auctions and second-price auctions, and when homogeneously risk averse bidders endogenously enter one of the auctions. In order to study this, we first consider bidders' entry decisions between exogenously given auctions. We find that there exists a symmetric entry equilibrium that is unique and is characterized by a mixed strategy, which depends on whether bidders exhibit constant, decreasing or increasing absolute risk aversion. In a next step, we endogenize the sellers' choice of auctions. We show that competing sellers have a dominant strategy to select first-price auctions if bidders exhibit nondecreasing absolute risk aversion. If bidders exhibit decreasing absolute risk aversion, other equilibria may exist in which sellers select second-price auctions as well. For instance, we demonstrate that sellers may select second-price auctions if the distribution of private values is sufficiently skewed.
\end{abstract}

Keywords Auctions · Endogenous entry · Risk aversion · Competing sellers JEL Classification D44 · D81

\begin{abstract}
We are grateful to Stephanie Rosenkranz, Britta Hoyer and Swantje Falcke for useful comments and suggestions. We would also like to thank the participants of the TKI Seminar Series in Utrecht, the Maastricht Lecture Series in Economics, the 2014 International Workshop on Game Theory and Economic Applications of the Game Theory Society in São Paulo, the 2015 Conference on Economic Design in Istanbul and the 2015 International Conference on Game Theory in Stony Brook. We thank Emesa Nederland BV for financial support. Special thanks are due to one anonymous referee and to the editor for helpful comments.
\end{abstract}

$凶$ Joyce Delnoij J.M.J.Delnoij@uu.nl

1 Department of Sociology/ICS, Utrecht University, P.O.Box 80140, 3508 TC Utrecht, The Netherlands

2 Utrecht University School of Economics, Utrecht University, P.O.Box 80125, 3508 TC Utrecht, The Netherlands 


\section{Introduction}

Nowadays, a vast array of commodities and services is being sold through online auctions. Online marketplaces like eBay offer a multitude of simultaneous auctions in which goods such as collectibles and phones are sold. Moreover, specialized online auction stores sell goods for which well-established markets already exist, such as holidays, concert tickets and computers. In this online environment, a single good is often sold through a variety of auction formats. Vintage cars, watches and jewelry, for instance, are sold through both English and Dutch auctions. ${ }^{1}$ This implies that sellers operate in a competitive market, in which they compete not only through the goods they sell, but also through the auction formats in which they sell these goods.

The economics literature has long treated auctions as isolated, studying how a single seller facing multiple bidders can maximize his revenue or, in the case of procurement auctions, how a single bidder facing multiple sellers can maximize her utility. Auction theory's most celebrated results, the revenue ranking theorems, compare the expected revenues of different auction formats while treating the number of bidders in each auction as given (e.g., Vickrey 1961; Myerson 1981; Riley and Samuelson 1981; Maskin and Riley 1984). Though these results have proved to be very valuable for the design of auctions for isolated sales such as the spectrum auctions, the traditional revenue ranking theorems may no longer apply if sellers operate in a competitive market, where the ability to attract bidders is a crucial determinant of an auction's success (e.g., Klemperer 2002; Ivanova-Stenzel and Salmon 2008a). After all, an auction that in isolation generates the highest revenue may no longer do so if bidders have no incentive to enter this auction. "In practice, auctions [...] often fail because of insufficient interest by bidders" (Milgrom 2004, p. 209). A seller operating in a competitive market should therefore consider bidders' preferences, as well as the selling mechanisms his competitors offer, when deciding which auction to offer.

The aim of this paper is to study the auction selection problem of competing sellers. That is, we theoretically investigate which auctions are selected by sellers when they operate in a competitive market and when bidders endogenously enter auctions. In doing so, we consider an auction selection game consisting of three stages. At Stage 1 of the game, sellers decide which auctions to offer. At Stage 2, the bidders learn which auctions are offered and enter one of them. At Stage 3, the auctions are conducted.

Throughout this paper, we make the following assumptions about sellers. We consider risk neutral sellers who simultaneously offer a single unit of a homogeneous good in sealed bid auctions. More precisely, sellers may choose to offer a first-price auction or a second-price auction. These auctions and their dynamic counterparts (the Dutch and English auction, respectively) are frequently used both on and off the Internet and have, for that reason, also attracted considerable attention in the theoretical and experimental literature.

On the bidders' side, we assume that bidders demand one unit of the good and choose to enter one of the auctions. They cannot choose to opt out of the auctions or enter multiple auctions instead. We assume that bidders have independent private values,

\footnotetext{
1 For example, see www.catawiki.com for online ascending auctions and www.dutchauction.com for online descending auctions (both visited in June 2018). Both online auction platforms offer specialty objects such as fine arts and collector's items.
} 
but also consider an extension with affiliated private values (Sect. 6). Additionally, the bidders are ex ante symmetrically informed, which implies that they cannot make their entry decisions dependent on any private information they may have. Before entering an auction, bidders do not know their own value for the good, but they do know the distribution of values. This is a common assumption in much of the theoretical and experimental literature studying entry into auctions (e.g., McAfee and McMillan 1987b; Engelbrecht-Wiggans 1987, 1993; Levin and Smith 1994; Smith and Levin 1996; Pevnitskaya 2004; Palfrey and Pevnitskaya 2008; Ivanova-Stenzel and Salmon 2004a, b, 2008a, b). We also consider an extension where bidders know their values before entering (see Sect. 5.2). Furthermore, we assume that bidders know whether and to which extent they are risk averse, and assume that bidders are homogeneous in this respect. In an extension, we discuss the implications of allowing bidders to be heterogeneously risk averse (see Sect. 5.1).

Various studies have analyzed the role of risk aversion in auctions and have shown that it is a critical factor explaining why bidders may not be indifferent between various auction formats. Theoretical research predicts that risk aversion results in overbidding in first-price auctions but does not change the equilibrium bidding strategy in secondprice auctions (e.g., Riley and Samuelson 1981; Maskin and Riley 1984; Cox et al. 1985 , 1988). As such, it also affects the utility bidders can expect from participating in these auctions (Matthews 1983, 1987). Previous experimental studies have shown that risk aversion may play a role in bidders' entry decisions between different auctions formats, although the results are contingent on the experimental design. ${ }^{2}$ Our study aims to provide the theoretical foundations for these findings and additionally explores the implications for the auction selection problem of competing sellers.

The auction selection game is solved using backward induction. We use existing results on bidding strategies and bidder preferences among auctions to analyze bidders' entry decisions in Stage 2. In doing so, we extend the models of endogenous entry of Levin and Smith (1994), Smith and Levin (1996) and Pevnitskaya (2004), who model entry as a symmetric equilibrium involving mixed strategies. We find that the probability of entering each auction depends on whether bidders exhibit constant, decreasing or increasing absolute risk aversion. More specifically, when bidders decide between entering first-price and second-price auctions, all auctions are entered with equal probability if bidders are risk neutral or exhibit constant absolute risk aversion. However, if bidders exhibit decreasing absolute risk aversion, they are more likely to enter second-price auctions; if bidders exhibit increasing absolute risk aversion, they are more likely to enter first-price auctions. As risk averse bidders overbid in first-price auctions, but not in second-price auctions, these findings imply that in Stage 1 all sellers prefer to offer first-price auctions when bidders exhibit nondecreasing absolute risk aversion. However, when bidders exhibit decreasing absolute risk aversion, other auction selection equilibria may exist.

Our study adds to the literature on bidder preferences over auction formats and endogenous entry, as well as to the literature on competing auctions. Whereas auction

\footnotetext{
${ }^{2}$ Ivanova-Stenzel and Salmon (2004a, 2008b) find that risk aversion explains entry decisions between English and first-price auctions when each auction consists of only two bidders. In another set of experiments, the authors allow bidders to coordinate freely over the auctions (Ivanova-Stenzel and Salmon 2008a, 2011). In these circumstances, risk aversion does not seem to explain entry decisions.
} 
theorists have traditionally focused on the seller's perspective, researchers are now also taking the bidder's point of view. It can be seen that Myerson's (1981) proof for the revenue equivalence between first-price and second-price auctions follows from utility equivalence for risk neutral bidders. Risk neutral bidders are thus indifferent between first-price and second-price auctions. Matthews (1983, 1987) compares the utility of bidders with constant, decreasing and increasing absolute risk aversion. He finds that bidders who exhibit constant absolute risk aversion are also indifferent between first-price and second-price auctions. This result is later generalized by Monderer and Tennenholtz (2004) for all $k$-price auctions and by Hon-Snir (2005) for all auctions in which the bidder with the highest bid wins. Hon-Snir additionally shows that the utility equivalence for risk averse bidders holds if and only if bidders exhibit constant absolute risk aversion. This is consistent with the findings of Matthews (1987), who shows that bidders with decreasing absolute risk aversion prefer second-price auctions and bidders with increasing absolute risk aversion prefer first-price auctions.

The theoretical literature on entry into auctions studies the decision whether or not to enter an auction with an entry fee or when there exists an outside option. This literature can roughly be divided into two strands. The first strand assumes that bidders do not possess any private information before deciding to enter an auction or not. In this case, the theoretical literature focuses on two types of equilibria. McAfee and McMillan (1987b) and Engelbrecht-Wiggans (1987, 1993) focus on deterministic, asymmetric equilibria involving pure entry strategies. This approach results in a plethora of equilibria, where a subset of bidders enters the auction and another subset does not. The process by which symmetric bidders are divided into these subsets, however, is not identified. Levin and Smith (1994) and Smith and Levin (1996) therefore focus on a unique, stochastic, symmetric equilibrium involving mixed entry strategies. Various experimental and empirical studies have compared these two approaches and find that entry is best explained by the stochastic model (e.g., Smith and Levin 2002; Bajari and Hortacsu 2003; Reiley 2005). The second strand of the literature assumes that bidders obtain some type of private information before making their entry decisions. This includes bidders' private values (Menezes and Monteiro 2000) and bidders' heterogeneous rates of risk aversion (Pevnitskaya 2004; Palfrey and Pevnitskaya 2008). These studies find that there is a unique entry equilibrium in pure strategies, which involves a cutoff value based on the bidders' private information. To the best of our knowledge, our study is the first to develop a theoretical model on entry decisions between different auction formats, although some experimental studies on this topic exist (Ivanova-Stenzel and Salmon 2004a, b, 2008a, b, 2011; Engelbrecht-Wiggans and Katok 2005). ${ }^{3}$

Most studies in the competing auctions literature analyze auction selection problems where the dimension along which sellers compete is the reserve price or the entry fee (e.g., McAfee 1993; Peters and Severinov 1997; Damianov 2012). Instead, the dimension along which sellers compete in our study is the auction format itself. The study that is perhaps most closely related to ours is that of Monderer and Tennenholtz (2004), who theoretically investigate auction selection with bidders who exhibit con-

\footnotetext{
3 Extensive overviews of the literature on entry into auctions can be found in Kagel and Levin (2014) and Aycinena et al. (2018).
} 
stant absolute risk attitudes, but who assume exogenous random participation (McAfee and McMillan 1987a). They find that sellers prefer to select a first-price auction when bidders exhibit constant absolute risk aversion. When bidders exhibit constant absolute risk seekingness, however, sellers will be better off selecting a $k$-price auction of higher order. Including a larger range of risk attitudes and assuming entry to be stochastic allow us to obtain novel insights into auction selection and simultaneously add to existing revenue ranking results. Moreover, our model can be used to study auction versioning by monopolists as well (see "Appendix 3").

The remainder of this paper is structured as follows. Section 2 describes the model in detail. Section 3 analyzes the entry decisions in Stage 2 of our three-stage game, and Sect. 4 analyzes the auction selection in Stage 1. Section 5 considers extensions toward heterogeneous bidders, and Sect. 6 relaxes the assumption of independent private values. Finally, Sect. 7 discusses our findings and provides concluding remarks.

\section{Model}

Suppose that $M \geq 2$ sellers simultaneously each offer a single unit of a homogeneous good to a group of $N \geq 2$ bidders. Each seller decides to offer the good either in a first-price auction (FPA) or in a second-price auction (SPA); bidders are free to enter any auction. We assume that sellers are risk neutral and have zero value for the good. Bidder $i$ 's preferences are given by the utility function $u\left(x_{i}\right)$, which is twice continuously differentiable, satisfies $u^{\prime}\left(x_{i}\right)>0$ and $u^{\prime \prime}\left(x_{i}\right) \leq 0$, and where $x_{i}$ represents her payoff. Throughout this paper, we use $r$ to refer to the Arrow-Pratt coefficient of absolute risk aversion, such that $r=-u^{\prime \prime}\left(x_{i}\right) / u^{\prime}\left(x_{i}\right)$. We distinguish between constant $\left(\partial r / \partial x_{i}=0\right)$, decreasing $\left(\partial r / \partial x_{i}<0\right)$ and increasing $\left(\partial r / \partial x_{i}>0\right)$ absolute risk aversions. This is generally interpreted as how absolute risk aversion changes as an individual's wealth changes. In the context of auctions, however, this is interpreted as how absolute risk aversion changes as a bidder's value changes. Hence, decreasing absolute risk aversion (DARA) means that bidders take more risk as their value increases; increasing absolute risk aversion (IARA) means that bidders take less risk as their value increases; and constant absolute risk aversion (CARA) means that bidders take the same amount of risk as their value increases (see Sect. 4.1 for a further discussion regarding DARA).

We consider the following three-stage game, which is an extension of the models of endogenous entry by Levin and Smith (1994), Smith and Levin (1996), and Pevnitskaya (2004). At Stage 1 , seller $\ell \in\{1, \ldots, M\}$ selects auction format $a_{\ell} \in\{I, I I\}$, where $a_{\ell}$ refers to the auction format selected by seller $\ell$, which can take on one of the two values: $I$, referring to FPA, and $I I$, referring to SPA. At this stage, the number of bidders, $N$, their utility functions, $u\left(x_{i}\right)$, and the distribution of values, $F$, are common knowledge. Prior to Stage 2 , the $N$ bidders learn each $a_{\ell}$, i.e., they learn which auctions have been selected by the sellers. Subsequently, each of the $N$ bidders enters one of the auctions, such that $n_{\ell}$ bidders enter $a_{\ell}$, where $\sum_{\ell=1}^{M} n_{\ell}=N .{ }^{4}$ At the beginning of Stage 3, each bidder $i$ learns $n_{\ell}$ in the auction $a_{\ell}$ that she has entered. Moreover,

\footnotetext{
4 Note that which bidder enters which auction may be the result of mixed strategies.
} 
she receives her private value $v_{i}$, which is independently and identically distributed according to the common distribution function $F$, with strictly positive density $f$ on the interval $[\underline{v}, \bar{v}]$. All bidders then simultaneously submit sealed bids according to the unique, symmetric and increasing Bayesian Nash equilibrium bidding function $b\left(v \mid a_{\ell}, n_{\ell}\right)$ of the Stage 3 subgames. The outcome of the auctions is to allocate the goods to the highest bidders. If bidder $i$ wins the auction, she receives a payoff of $v_{i}-p_{i}$, where $p_{i}$ represents $i$ 's payment. Whereas in the FPA $p_{i}$ is equal to $i$ 's own bid, in the SPA it is equal to the bid of the second highest bidder. If bidder $i$ does not win the auction, she receives a payoff of zero.

The equilibria of the Stage 3 subgames have been extensively analyzed in the literature (e.g., Vickrey 1961; Riley and Samuelson 1981; Maskin and Riley 1984). In the FPA, the equilibrium bidding strategy when bidders are risk neutral is to bid an amount equal to the expectation of the highest of $n_{\ell}-1$ values below one's own value. When bidders are risk averse, however, the equilibrium bidding strategy is higher. In the SPA, the equilibrium bidding strategy is to bid one's own value, regardless of whether bidders are risk averse or not. Applying backward induction, we use these outcomes to analyze the entry decisions in Stage 2 and the selection of auctions in Stage 1 .

\section{Endogenous entry}

In this section, we analyze bidders' entry decisions in any Stage 2 subgame, where in line with backward induction bidders anticipate the unique, symmetric Bayesian Nash equilibrium of each Stage 3 subgame. Let $E\left[u \mid a_{\ell}, n_{\ell}\right]$ denote each bidder's ex ante expected utility in auction $a_{\ell}$, after learning $n_{\ell}$ and her value $v$, and bidding according to the symmetric equilibrium bidding strategy $b\left(v \mid a_{\ell}, n_{\ell}\right)$. Note that $E\left[u \mid a_{\ell}, n_{\ell}\right]$ is strictly decreasing in $n_{\ell}$, because an increase in the number of bidders decreases the probability of winning and raises the payment in both the FPA and the SPA (Smith and Levin 1996). Moreover, if a bidder is the only one in an auction, she will earn a positive payoff with certainty.

Following Levin and Smith (1994), Smith and Levin (1996) and Pevnitskaya (2004), we focus on a symmetric entry equilibrium of a Stage 2 subgame (henceforth referred to as symmetric entry equilibrium). Any symmetric entry equilibrium will necessarily involve mixed strategies, such that in equilibrium each bidder enters auction $a_{\ell}$ with probability $q_{\ell}^{*}$, where $\sum_{\ell=1}^{M} q_{\ell}^{*}=1$. The reason for this is simple. Suppose that no bidder enters auction $a_{\ell}$. Then each bidder has an incentive to switch to auction $a_{\ell}$, as in this auction she will be the only bidder and therefore earn a positive payoff with certainty. It follows that each auction must be chosen with positive probability. In equilibrium, each bidder must therefore be indifferent between entering auction $a_{\ell}$ and any other auction $a_{\ell^{\prime}}$. 
This implies that each bidder's ex ante expected utility of entering auction $a_{\ell}$ is described by:

$$
\sum_{n_{\ell}=1}^{N}\left(\begin{array}{l}
N-1 \\
n_{\ell}-1
\end{array}\right)\left(q_{\ell}^{*}\right)^{n_{\ell}-1}\left(1-q_{\ell}^{*}\right)^{N-n_{\ell}} E\left[u \mid a_{\ell}, n_{\ell}\right]
$$

where $q_{\ell}^{*} \in(0,1)$ and where the coefficient of $E\left[u \mid a_{\ell}, n_{\ell}\right]$ gives the binomial probability that exactly $n_{\ell}-1$ competing bidders also enter the auction, giving $n_{\ell}$ bidders in total. By summing over any possible $n_{\ell}$ ranging from 1 to $N$, each bidder considers every possible number of competing bidders in the chosen auction. ${ }^{5,6}$

The value of the equilibrium probability of entering auction $a_{\ell}$, given by $q_{\ell}^{*}$, crucially depends on the auction formats that are selected by the sellers and on the utility functions of the bidders. Lemma 1 defines $q_{\ell}^{*}$ when all or some sellers select the same auction format.

Lemma 1 If there exist sellers $\ell$ and $\ell^{\prime}$ who select the same auction format $\left(a_{\ell}=a_{\ell^{\prime}}\right)$, then in any symmetric entry equilibrium $q_{\ell}^{*}=q_{\ell^{\prime}}^{*}$.

Proof Let $a_{\ell}=a_{\ell^{\prime}}$. It follows immediately that $E\left[u \mid a_{\ell}, n_{\ell}\right]=E\left[u \mid a_{\ell^{\prime}}, n_{\ell^{\prime}}\right]$ for $n_{\ell}=$ $n_{\ell^{\prime}}$. Further note that (1) is a polynomial in Bernstein form (e.g., Farouki 2012). By Lemma 5 in "Appendix 1" and because $E\left[u \mid a_{\ell}, n_{\ell}\right]$ is strictly decreasing in $n_{\ell}$, it follows that (1) is continuous and strictly decreasing in $q_{\ell}$. Hence, the ex ante expected utility of entering auction $a_{\ell}$ equals that of any other auction $a_{\ell^{\prime}}$ if and only if $q_{\ell}^{*}=q_{\ell^{\prime}}^{*}$.

From Lemma 1, it follows that when more than one seller selects the FPA (or, equivalently, selects the SPA), then the ex ante expected utility in any of those identical auction formats is the same as long as the number of bidders in each auction is also the same. This implies that bidders are indifferent between entering any auction of the same format, and will enter any such auction with the same probability. Lemma 1 further implies that when all sellers select FPAs or, equivalently, SPAs, bidders will enter each auction with equal probability in equilibrium, that is, $q_{\ell}^{*}=(1 / M)$.

Let us now consider a case where sellers offer their respective goods in different auction formats. Suppose that $m_{I} \geq 1$ sellers offer an FPA and $m_{I I} \geq 1$ sellers offer an SPA, where $m_{I}+m_{I I}=M$. By Lemma 1, any auction of the same format is entered with the same probability. As such, we can view each bidder's entry decision as consisting of two stages. First, the bidder chooses between entering an FPA and an SPA. Second, given this choice, she will choose to enter any of the FPAs, or SPAs, with equal

\footnotetext{
5 Throughout the paper, we will refer to $E\left[u \mid a_{\ell}, n_{\ell}\right]$ as the ex ante expected utility in a specific auction and to (1) as the ex ante expected utility of entering a specific auction.

${ }^{6}$ Like in the case where bidders choose between entering a single auction and not entering it (e.g., Levin and Smith 1994; Smith and Levin 1996), even with symmetric bidders asymmetric entry equilibria may exist, e.g., where bidders partition themselves over auctions such that each bidder enters a specific auction with probability 1 . Furthermore, note that the assumption of pure strategies may lead to very many equilibria, dependent on how bidders partition themselves over the auctions. This creates an equilibrium selection problem. Therefore, we solely focus on a symmetric entry equilibrium, which not only restores full symmetry to the model but also turns out to be unique.
} 
probability. Therefore, with a slight abuse of notation, let us denote the probability of entering an FPA by $q$ and entering an SPA by $(1-q)$. Then the probability of entering one of the FPAs, that is entering an auction $a_{\ell}=I$, is equal to $q / m_{I}$. Correspondingly, the probability of entering one of the SPAs, that is entering an auction $a_{\ell}=I I$, is equal to $(1-q) / m_{I I}$. In this way, $m_{I}\left(q / m_{I}\right)+m_{I I}\left((1-q) / m_{I I}\right)=1$. Hence, our symmetric entry equilibrium can be defined by a single $q^{*}$.

The symmetry from Lemma 1 implies that, for $q^{*} \in(0,1)$ to constitute a symmetric entry equilibrium, it now suffices that each bidder is indifferent between one of the FPAs and one of the SPAs. That is, any symmetric entry equilibrium is described by:

$$
\begin{aligned}
& \sum_{n_{I}=1}^{N}\left(\begin{array}{c}
N-1 \\
n_{I}-1
\end{array}\right)\left(\frac{q^{*}}{m_{I}}\right)^{n_{I}-1}\left(1-\frac{q^{*}}{m_{I}}\right)^{N-n_{I}} E\left[u \mid I, n_{I}\right] \\
& =\sum_{n_{I I}=1}^{N}\left(\begin{array}{c}
N-1 \\
n_{I I}-1
\end{array}\right)\left(\frac{1-q^{*}}{m_{I I}}\right)^{n_{I I}-1}\left(1-\frac{1-q^{*}}{m_{I I}}\right)^{N-n_{I I}} E\left[u \mid I I, n_{I I}\right]
\end{aligned}
$$

where the left-hand side (LHS) of (2) gives the ex ante expected utility of entering a specific FPA and the right-hand side (RHS) gives the ex ante expected utility of entering a specific SPA, and where $n_{I}-1$ denotes the number of competing bidders entering an FPA and $n_{I I}-1$ denotes the number of competing bidders entering an SPA. We find that the resulting equilibrium probability of entry is unique for a given $r{ }^{7}$

Lemma 2 Suppose that bidders may choose between entering at least one FPA and at least one SPA. Then there exists a symmetric entry equilibrium in mixed strategies, such that each bidder enters an FPA with probability $q^{*}$ and enters an SPA with probability $1-q^{*}$. The equilibrium probability of entry is implicitly defined by (2) and is unique for a given absolute rate of risk aversion $r$.

Proof Define $z(q, r)$ as the function equal to the LHS minus the RHS of (2). As both the LHS and the RHS are polynomials in Bernstein form, and because $E\left[u \mid a_{\ell}, n_{\ell}\right]$ is strictly decreasing in $n_{\ell}$, we can again use Lemma 5 in "Appendix 1" to show that the LHS of (2) is continuous and strictly decreasing in $q$, and that the RHS of (2) is continuous and strictly increasing in $q$. This implies that $z(q, r)$ is continuous and strictly decreasing in $q$.

Equilibrium is achieved when $z\left(q^{*}, r\right)=0$. Notice that any $q^{*}$ satisfying this condition must be in the interval $(0,1)$. For instance, suppose that $q^{*}=0$, such that all bidders enter an SPA. Then $z\left(q^{*}, r\right)>0$ and each bidder can receive a positive payoff with certainty by entering an FPA. Conversely, suppose that $q^{*}=1$, such that all bidders enter an FPA. Then $z\left(q^{*}, r\right)<0$ and each bidder can receive a positive payoff with certainty by entering an SPA. As a result, only $0<q^{*}<1$ can satisfy

\footnotetext{
7 Note that the actual number of bidders in a specific FPA then follows a binomial distribution with mean $\left(q^{*} / m_{I}\right) N$ and variance $\left(q^{*} / m_{I}\right)\left[1-\left(q^{*} / m_{I}\right)\right] N$. Similarly, the actual number of bidders in a specific SPA follows a binomial distribution with mean $\left[\left(1-q^{*}\right) / m_{I I}\right] N$ and variance $\left[\left(1-q^{*}\right) / m_{I I}\right]\{1-[(1-$ $\left.\left.\left.q^{*}\right) / m_{I I}\right]\right\} N$.
} 
$z\left(q^{*}, r\right)=0$. By the intermediate value theorem, it then follows that there exists a unique symmetric equilibrium probability of entry, $q^{*}$, and it is defined by (2).

While the symmetric entry equilibrium is defined by a single $q^{*}$, the value of $q^{*}$ itself differs with the absolute rate of risk aversion $r$. Lemma 3 and Proposition 1 give $q^{*}$ for different circumstances.

Lemma 3 Suppose that bidders may choose between entering at least one FPA and at least one SPA, and that bidders are risk neutral $(r=0)$. The symmetric entry equilibrium is then given by $q^{*}=\left(m_{I} / M\right)$, where $q^{*}$ defines the equilibrium probability of entering an FPA and $1-q^{*}$ defines the equilibrium probability of entering an SPA, and where $M$ denotes the total number of auctions offered, consisting of $m_{I}$ FPAs and $m_{I I}$ SPAs.

Proof Let $r=0$. From the utility equivalence principle for risk neutral bidders that follows from Myerson (1981), we know that $E\left[u \mid I, n_{I}\right]=E\left[u \mid I I, n_{I I}\right]$ for $n_{I}=n_{I I}$. As a result, each bidder's entry decision is only affected by the number of competing bidders in each auction. This leads bidders to randomize over auctions with equal probability, that is, $q^{*}=\left(m_{I} / M\right)$.

Lemma 3 therefore shows that when sellers select both FPAs and SPAs and bidders are risk neutral, then bidders will enter each auction with equal probability. When bidders are risk averse, however, the equilibrium probability of entry depends on whether bidders exhibit constant, decreasing or increasing absolute risk aversion.

Proposition 1 Suppose that bidders may choose between entering at least one FPA and at least one SPA, and that bidders are risk averse $(r>0)$. The symmetric entry equilibrium is then given by

(i) $q^{*}=\left(m_{I} / M\right)$, if bidders exhibit CARA

(ii) $q^{*}<\left(m_{I} / M\right)$, if bidders exhibit DARA

(iii) $q^{*}>\left(m_{I} / M\right)$, if bidders exhibit IARA

where $q^{*}$ defines the equilibrium probability of entering an FPA and $1-q^{*}$ defines the equilibrium probability of entering an SPA, and where $M$ denotes the total number of auctions offered, consisting of $m_{I}$ FPAs and $m_{I I}$ SPAs.

Proof The proof of this proposition consists of two steps. Recall that the value of $q^{*}$ that satisfies $z\left(q^{*}, r\right)=0$ characterizes the symmetric entry equilibrium. In Step 1, we show that if each auction is entered with equal probability, i.e., $q=\left(m_{I} / M\right)$, then $z(q, r)$ is equal to zero if bidders exhibit CARA, is negative if bidders exhibit DARA, and is positive if bidders exhibit IARA. In Step 2 of the proof, we demonstrate how $q$ needs to be adjusted such that the equilibrium condition is satisfied.

Step 1 Suppose that $r>0$ and that $q=\left(m_{I} / M\right)$. From Theorem 1 of Matthews (1987, p. 638), it then follows that, for $n_{I}=n_{I I}$, the ex ante expected utility in each auction is

(i) $E\left[u \mid I, n_{I}\right]=E\left[u \mid I I, n_{I I}\right]$, if bidders exhibit CARA

(ii) $E\left[u \mid I, n_{I}\right]<E\left[u \mid I I, n_{I I}\right]$, if bidders exhibit DARA 
(iii) $E\left[u \mid I, n_{I}\right]>E\left[u \mid I I, n_{I I}\right]$, if bidders exhibit IARA

This implies that, for a given $q=\left(m_{I} / M\right)$, the LHS of (2) is equal to the RHS if bidders exhibit CARA, is smaller than the RHS if bidders exhibit DARA, and is larger than the RHS if bidders exhibit IARA. Hence,

(i) $z\left(\left(m_{I} / M\right), r\right)=0$, if bidders exhibit CARA

(ii) $z\left(\left(m_{I} / M\right), r\right)<0$, if bidders exhibit DARA

(iii) $z\left(\left(m_{I} / M\right), r\right)>0$, if bidders exhibit IARA

Step 2 Part (i) of this proposition follows immediately from Lemma 2 . What follows here is a proof of part (ii). From the proof of Lemma 2, we know that $z(q, r)$ is continuous and strictly decreasing in $q$. As $z\left(\left(m_{I} / M\right), r\right)<0$ if bidders exhibit DARA, it therefore follows that $q$ needs to decrease in order to achieve equilibrium. As a result, $q^{*}<\left(m_{I} / M\right)$ if bidders exhibit DARA. Part (iii) of this proposition is proved analogously.

Proposition 1 implies that if bidders exhibit CARA, they will enter each auction with equal probability. However, bidders will be more likely to enter an SPA if they exhibit DARA, and will be more likely to enter an FPA if they exhibit IARA. These findings follow from the utility equivalence results from Matthews (1983, 1987), who compares auctions for risk averse bidders when the number of bidders in each auction is fixed. Risk averse bidders tend to bid more in the FPA than in the SPA, making the SPA more desirable from the bidders' perspective. At the same time, however, the payment in the SPA is a random variable, making the FPA more desirable. Matthews (1987) finds that when bidders exhibit CARA, the fact that bids are higher in the FPA exactly compensates the fact that the payment in the SPA is riskier, so that bidders are indifferent between the two auction formats. However, if bidders exhibit DARA, they prefer the SPA to the FPA, while the opposite is true if they exhibit IARA.

The envelope theorem is the key to understanding the findings of Matthews (1987). By this theorem, when marginally changing a parameter in the bidder's utility function, we need only look at the direct effect of this parameter on the utility, and not at the indirect effect that the parameter has through a change in the optimal bidding function. Given that the bidder with the lowest value $\underline{v}$ is indifferent between the FPA and the SPA, one can now check how a marginal increase in the value affects the bidder's risk attitudes toward the FPA and the SPA, considered as given lotteries. For IARA, marginally increasing the value means that the bidder becomes more risk averse, and therefore is more inclined toward the less risky auction, namely the FPA. For DARA, marginally increasing the value means that the bidder becomes less risk averse, and therefore more inclined toward the riskier auction, namely the SPA. For CARA, as increasing her value does not change her risk attitudes, she continues to be indifferent between the FPA and the SPA.

Combining Matthews's (1987) findings with the fact that the ex ante expected utility in an auction is decreasing in the number of bidders gives us Proposition 1. For instance, suppose that bidders exhibit DARA. In this case, each bidder is only indifferent between entering an FPA and an SPA when the number of competing bidders is larger in the SPA than in the FPA. Similarly, if bidders exhibit IARA, each bidder is only indifferent between entering an FPA and an SPA when the number of competing bidders is larger in the FPA than in the SPA. 
Simulations for $M=2$ and $m_{I}=m_{I I}=1$ with utility functions exhibiting CARA, DARA or IARA show that $q^{*}$ remains close to 0.5 for any absolute rate of risk aversion $r$. This is shown in Figs. 1, 2 and 3 in Sect. 4, which show how $q^{*}$ develops when bidders exhibiting DARA get more risk averse. ${ }^{8}$ It seems that even though bidders may highly prefer one auction over the other, there are negative externalities from other bidders entering the auction. This latter effect seems to be rather strong, causing $q^{*}$ to remain close to 0.5 even when bidders have a strong preference for one of the auction formats.

\section{Auction selection}

In this section, we use the insights obtained in Sect. 3 to evaluate the sellers' decisions in Stage 1 of our game, where consistent with backward induction, sellers anticipate the unique, symmetric entry equilibrium of each Stage 2 subgame and the unique, symmetric Bayesian Nash equilibrium of each Stage 3 subgame. Recall that there are $M$ sellers, who each offer one unit of a homogeneous good in either an FPA or an SPA. Like before, let $q^{*}$ denote the equilibrium probability of entering an FPA and $1-q^{*}$ the equilibrium probability of entering an SPA. The expected revenues of offering an FPA or an SPA are then given by:

$$
\begin{aligned}
& E\left[R_{I}\right]=\sum_{n_{I}=0}^{N}\left(\begin{array}{c}
N \\
n_{I}
\end{array}\right)\left(\frac{q^{*}}{m_{I}}\right)^{n_{I}}\left(1-\frac{q^{*}}{m_{I}}\right)^{N-n_{I}} R_{I}\left(n_{I}, r\right) \\
& E\left[R_{I I}\right]=\sum_{n_{I I}=0}^{N}\left(\begin{array}{c}
N \\
n_{I I}
\end{array}\right)\left(\frac{1-q^{*}}{m_{I I}}\right)^{n_{I I}}\left(1-\frac{1-q^{*}}{m_{I I}}\right)^{N-n_{I I}} R_{I I}\left(n_{I I}\right)
\end{aligned}
$$

where $R_{I}\left(n_{I}, r\right)$ is the seller's ex ante expected revenue when the FPA is entered by $n_{I}$ bidders who have absolute rate of risk aversion $r$. It represents the expected payment made by the highest of $n_{I}$ bidders. Similarly, $R_{I I}\left(n_{I I}\right)$ is the seller's ex ante expected revenue when the SPA is entered by $n_{I I}$ bidders. The ex ante expected revenues of both auctions are increasing in the number of bidders, $n_{I}$ and $n_{I I}$ (e.g., Kagel and Levin 1993).

The revenue equivalence theorem states that the ex ante expected revenue from the FPA equals that of the SPA if bidders are risk neutral, that is, $R_{I}\left(n_{I}, 0\right)=R_{I I}\left(n_{I I}\right)$ for $n_{I}=n_{I I}$ (Vickrey 1961). Recall that, in equilibrium, bidders enter each auction with equal probability, i.e., $q^{*}=\left(m_{I} / M\right)$, if they are risk neutral (see Lemma 3$)$. Hence, it immediately follows that $E\left[R_{I}\right]=E\left[R_{I I}\right]$ if bidders are risk neutral, and therefore, competing sellers will be indifferent between selecting the FPA and the SPA.

If bidders are risk averse, the situation is more complex. Whereas the equilibrium bidding strategy in the SPA is insensitive to changes in risk attitudes, the equilibrium bidding strategy in the FPA is increasing in risk aversion (e.g., Riley and Samuelson 1981; Maskin and Riley 1984; Cox et al. 1985, 1988). As a result, the ex ante expected

\footnotetext{
8 As DARA is implied by constant relative risk aversion, we focus on the effect of different levels of relative risk aversion in our simulations underlying Figs. 1, 2 and 3.
} 
revenue of the FPA is larger than that of the SPA if bidders are risk averse, that is, $R_{I}\left(n_{I}, r\right)>R_{I I}\left(n_{I I}\right)$ for any $n_{I}=n_{I I}$ and $r>0$. Given our results from Sect. 3 , this implies the following for the expected revenues when bidders exhibit nondecreasing absolute risk aversion (NDARA).

Lemma 4 Suppose that there exist sellers $\ell$ and $\ell^{\prime}$ such that $\ell$ selects the FPA $\left(a_{\ell}=I\right)$ and $\ell^{\prime}$ selects the SPA $\left(a_{\ell^{\prime}}=I I\right)$, and that bidders are risk averse $(r>0)$, exhibit NDARA and choose between auctions as described in Proposition 1. The FPA then yields more expected revenue than the SPA.

Proof Proposition 1 shows that $q^{*} \geq\left(m_{I} / M\right)$ if bidders exhibit CARA or IARA, where $q^{*}$ defines the equilibrium probability of entering an FPA and $1-q^{*}$ defines the equilibrium probability of entering an SPA. This permits direct comparison of expected revenues.

$$
\begin{aligned}
E\left[R_{I}\right] & =\sum_{n_{I}=0}^{N}\left(\begin{array}{c}
N \\
n_{I}
\end{array}\right)\left(\frac{q^{*}}{m_{I}}\right)^{n_{I}}\left(1-\frac{q^{*}}{m_{I}}\right)^{N-n_{I}} R_{I}\left(n_{I}, r\right) \\
& >\sum_{n_{I I}=0}^{N}\left(\begin{array}{c}
N \\
n_{I I}
\end{array}\right)\left(\frac{q^{*}}{m_{I}}\right)^{n_{I I}}\left(1-\frac{q^{*}}{m_{I}}\right)^{N-n_{I I}} R_{I I}\left(n_{I I}\right) \\
& \geq \sum_{n_{I I}=0}^{N}\left(\begin{array}{c}
N \\
n_{I I}
\end{array}\right)\left(\frac{1-q^{*}}{m_{I I}}\right)^{n_{I I}}\left(1-\frac{1-q^{*}}{m_{I I}}\right)^{N-n_{I I}} R_{I I}\left(n_{I I}\right)=E\left[R_{I I}\right]
\end{aligned}
$$

The strict inequality is based on the fact that $R_{I}\left(n_{I}, r\right)>R_{I I}\left(n_{I I}\right)$ for any $n_{I}=n_{I I}$ and $r>0$. To prove that the second inequality holds, note that both equations are polynomials in Bernstein form (e.g., Farouki 2012). Further recall that the ex ante expected revenue of the SPA is strictly increasing in the number of bidders. Then by Lemma 5 in "Appendix 1," the LHS of the second inequality is continuous and strictly increasing in $q$ and the RHS is continuous and strictly decreasing in $q$. Substituting $q^{*}=\left(m_{I} / M\right)$ and $m_{I I}=M-m_{I}$ shows that the two sides of the second inequality are equal for $q^{*}=\left(m_{I} / M\right)$. It then follows that the LHS of the second inequality is strictly greater than the RHS for any $q>\left(m_{I} / M\right)$, and vice versa for any $q<\left(m_{I} / M\right)$. Since $q^{*} \geq\left(m_{I} / M\right)$ if bidders exhibit CARA or IARA (see Proposition 1$)$, the second inequality must hold. This concludes the proof.

If competing sellers offer their respective goods in different auction formats, and risk averse bidders exhibiting NDARA endogenously enter one of the auctions, then each bidder is at least as likely to enter an FPA as she is likely to enter an SPA (see Proposition 1). This finding, combined with the familiar ranking of ex ante expected revenues for risk averse bidders, gives us Lemma 4. Our finding also implies that DARA is a necessary condition for the traditional revenue ranking to reverse. After all, if bidders exhibit DARA, they prefer the SPA over the FPA, which makes them more likely to enter the SPA. Only if sufficiently many bidders enter an SPA, the initial advantage of the FPA may be overcome. 
We now focus on the auction selection game in Stage 1, where competing sellers choose which auction format to offer while taking into account the bidding behavior and entry decisions of risk averse bidders. Recall that in the auction selection game each seller has two possible actions: to select an FPA or an SPA. The payoffs of selecting an FPA are given by (3); the payoffs of selecting an SPA are given by (4). Let us first point out that the strategy profile where all sellers select FPAs generates greater total payoffs to the sellers than the strategy profile where all sellers select SPAs. From Lemma 1, we know that when all sellers select the same auction, each auction is entered with equal probability. Hence, the payoffs for seller $\ell \in\{1,2, \ldots, M\}$ of selecting an FPA when all $M-1$ competing sellers also select an FPA are given by:

$$
\sum_{n_{I}=0}^{N}\left(\begin{array}{l}
N \\
n_{I}
\end{array}\right)\left(\frac{1}{M}\right)^{n_{I}}\left(\frac{M-1}{M}\right)^{N-n_{I}} R_{I}\left(n_{I}, r\right)
$$

Similarly, seller $\ell$ 's payoffs of selecting an SPA when all $M-1$ competing sellers also select an SPA are given by:

$$
\sum_{n_{I I}=0}^{N}\left(\begin{array}{c}
N \\
n_{I I}
\end{array}\right)\left(\frac{1}{M}\right)^{n_{I I}}\left(\frac{M-1}{M}\right)^{N-n_{I I}} R_{I I}\left(n_{I I}\right)
$$

As a result of the revenue ranking with risk averse bidders, the strategy profile where all sellers select an FPA gives greater total payoffs than the one where all sellers select an SPA. The ranking of the other strategy profiles is influenced by whether bidders exhibit CARA, DARA or IARA, as it crucially depends on the value of the equilibrium probability of entry, $q^{*}$.

Proposition 2 Suppose that the M competing sellers choose either an FPA or an SPA, and that bidders are risk averse $(r>0)$, exhibit NDARA and choose between auctions as described in Proposition 1. Then each seller has a dominant strategy to select the FPA. ${ }^{9}$

Proof Suppose that $m_{I I}$ sellers currently offer an SPA, with $1 \leq m_{I I} \leq M$. Then the auction of an individual seller offering an SPA is entered with probability $\left(1-q^{*}\right) / m_{I I}$, where $q^{*}$ denotes the equilibrium probability of entering an FPA (note that $q^{*}>0$ if $m_{I I}<M$, and $q^{*}=0$ if $m_{I I}=M$ ). Now suppose that one of the sellers switches to offering an FPA. This leads to a new symmetric entry equilibrium in the corresponding Stage 2 subgame, where the aforementioned seller's auction is now chosen with probability $q^{* \prime} /\left(M-m_{I I}+1\right)$, where $q^{* \prime}$ denotes the new equilibrium probability of choosing an FPA (note that $q^{* \prime}=1$ if $m_{I I}=1$, as in that case only FPAs are offered, and each identical FPA is entered with the same probability $(1 / M))$. We now claim that the individual seller's expected revenue will be greater when selecting the FPA than when selecting the SPA if bidders have NDARA utility functions. That is,

\footnotetext{
9 We are grateful to an anonymous referee for this formulation of the proposition.
} 


$$
\begin{aligned}
& \sum_{n_{I I}=0}^{N}\left(\begin{array}{c}
N \\
n_{I I}
\end{array}\right)\left(\frac{1-q^{*}}{m_{I I}}\right)^{n_{I I}}\left(1-\frac{1-q^{*}}{m_{I I}}\right)^{N-n_{I I}} R_{I I}\left(n_{I I}\right) \\
& \quad<\sum_{n_{I}=0}^{N}\left(\begin{array}{l}
N \\
n_{I}
\end{array}\right)\left(\frac{q^{* \prime}}{M-m_{I I}+1}\right)^{n_{I}}\left(1-\frac{q^{* \prime}}{M-m_{I I}+1}\right)^{N-n_{I}} R_{I}\left(n_{I}, r\right)
\end{aligned}
$$

To see why this is true, note that from the traditional revenue ranking it follows that $R_{I}\left(n_{I}, r\right)>R_{I I}\left(n_{I I}\right)$ for any $n_{I}=n_{I I}$ and $r>0$. By Proposition 1 , if bidders exhibit CARA, then $\left(1-q^{*}\right) / m_{I I}=q^{* \prime} /\left(M-m_{I I}+1\right)=(1 / M)$. As the distribution over all possible numbers of entering bidders is then identical in both auctions, revenue ranking implies that the FPA yields higher expected revenue. Also by Proposition 1, if bidders exhibit IARA, then $\left(1-q^{*}\right) / m_{I I}<(1 / M)<q^{* \prime} /\left(M-m_{I I}+1\right)$. Moreover, by the proof of Lemma 4 , the LHS and RHS are continuously increasing in respectively $\left(1-q^{*}\right) / m_{I I}$ and $q^{* \prime} /\left(M-m_{I I}+1\right)$. Starting now from the case where $\left(1-q^{*}\right) / m_{I I}=$ $q^{* \prime} /\left(M-m_{I I}+1\right)$, the fact that $\left(1-q^{*}\right) / m_{I I}<(1 / M)<q^{* \prime} /\left(M-m_{I I}+1\right)$ means that, all else equal, the RHS becomes smaller than the LHS. The inequality is further reinforced by the revenue ranking. This concludes the proof of this proposition.

Proposition 2 implies that if bidders exhibit NDARA, all competing sellers select an FPA. This follows naturally, as in these cases the FPA is ex ante (weakly) preferred to the SPA by both sellers and bidders. If bidders exhibit DARA, however, two opposing effects occur. On the one hand, the FPA generates more ex ante expected revenue than the SPA if bidders are risk averse. On the other hand, if bidders exhibit DARA, they are more likely to enter the SPA than the FPA, that is, $q^{*}<\left(m_{I} / M\right)$ by Proposition 1. Proposition 2 implies that DARA is a necessary condition for any equilibrium other than one where all sellers select an FPA to exist, but it is by itself not sufficient. In the remainder of this section, we demonstrate by example that if bidders exhibit DARA, other equilibria may exist in which sellers select SPAs as well.

\subsection{An example of auction selection with DARA bidders}

Consider the following example, where $M=2$ sellers compete to attract bidders, and where bidders' preferences are described by a particular form of DARA, i.e., the constant relative risk aversion (CRRA) utility function. ${ }^{10}$ That is, we assume that each bidder $i$ has a utility function of the form $u\left(x_{i}\right)=x_{i}^{(1-\rho)}$, where $x_{i}$ represents a bidder's payoff and $\rho \in[0,1)$ represents the coefficient of relative risk aversion. This utility function is often used in auction theory, as in combination with independent private values it results in a linear bidding function that captures bidding behavior rather well. Using parameterization of the above-mentioned utility function in experimental FPAs, estimates for the coefficient of relative risk aversion range from $\rho=0.67$ to $\rho=0.52$

\footnotetext{
10 For simplicity, we have chosen to present here the simulations for one of the simplest and most often used utility functions in economics: the power utility function for positive powers. However, qualitatively similar results can be obtained when using a more general utility function, for instance, one exhibiting hyperbolic absolute risk aversion. For a discussion of the characteristics of the power utility function, see Wakker (2008).
} 
and $\rho=0.48$ (Cox and Oaxaca 1996; Goeree et al. 2003; Chen and Plott 1998, respectively). Yet, to the best of our knowledge, no studies exist that systematically analyze whether bidders' preferences can indeed best be described by DARA utility functions, or whether perhaps CARA or IARA are more suitable assumptions in the context of auctions. Following Arrow's (1971) hypothesis, however, economists seem to agree that DARA is the more customary assumption. Moreover, DARA seems to best support the experimental evidence outside the auction realm (e.g., Levy 1994; Holt and Laury 2002).

Recall that if bidder $i$ wins the auction, her payoff $\left(x_{i}\right)$ is equal to her private value $\left(v_{i}\right)$ minus her payment $\left(p_{i}\right)$. If bidder $i$ loses the auction, her payoff is equal to zero. We assume that values are distributed according to $F=v^{\alpha}$ for $v \in[0,1]$, where $\alpha \geq 1$ and takes integer values only. Note that values are uniformly distributed if $\alpha=1$. An increase in $\alpha$ represents an increase in the skewness of the distribution of values such that higher values are drawn with larger probability. In this case, the ex ante expected revenues are given by:

$$
\begin{aligned}
R_{I}\left(n_{I}, r\right) & =\frac{\alpha\left(n_{I}-1\right)}{\alpha\left(n_{I}-1\right)+1-\rho} \frac{\alpha n_{I}}{\alpha n_{I}+1} \\
R_{I I}\left(n_{I I}\right) & =\frac{\alpha\left(n_{I I}-1\right)}{\alpha\left(n_{I I}-1\right)+1} \frac{\alpha n_{I I}}{\alpha n_{I I}+1}
\end{aligned}
$$

The bidders' ex ante expected utilities in the auctions are given by:

$$
\begin{aligned}
E\left[u \mid I, n_{I}\right] & =\frac{\alpha}{\alpha n_{I}+1-\rho}\left(\frac{1-\rho}{\alpha\left(n_{I}-1\right)+1-\rho}\right)^{1-\rho} \\
E\left[u \mid I I, n_{I I}\right] & =\frac{\alpha}{\alpha n_{I I}+1-\rho} \frac{\left(\alpha\left(n_{I I}-1\right)\right) !}{\left(\alpha\left(n_{I I}-1\right)+1-\rho\right) !}
\end{aligned}
$$

where $\left(\alpha\left(n_{I I}-1\right)+1-\rho\right) ! \equiv \prod_{i=1}^{\alpha\left(n_{I I}-1\right)}(i+1-\rho)$. The derivations of these results are shown in "Appendix 2 ". 11

To analyze which auctions are selected by competing sellers, we use (7) and (8) to compute the equilibrium probability of entry, $q^{*}$, and use (5) and (6) to compute $q$ and $\bar{q}$. Let $\underline{q}$ be defined as the probability of entry for which seller $\ell \in\{1,2\}$ is indifferent between selecting the FPA and the SPA given that seller $\ell^{\prime}$ offers an FPA. Recall that if both sellers offer the same auction format, bidders enter each auction with equal probability, i.e., $q^{*}=0.5$.

$$
0.5^{N} \sum_{n_{I}=0}^{N}\left(\begin{array}{c}
N \\
n_{I}
\end{array}\right) R_{I}\left(n_{I}, r\right)=\sum_{n_{I I}=0}^{N}\left(\begin{array}{c}
N \\
n_{I I}
\end{array}\right)(1-\underline{q})^{n_{I I}}(\underline{q})^{N-n_{I I}} R_{I I}\left(n_{I I}\right)
$$

$\overline{11}$ An alternative way of formulating $E\left[u \mid I I, n_{I I}\right]$ is as a function of the gamma function, $\Gamma$. In this case, it is given by

$$
E\left[u \mid I I, n_{I I}\right]=\frac{\alpha}{\alpha n_{I I}+1-\rho} \frac{\Gamma\left(\alpha\left(n_{I I}-1\right)+1\right) \Gamma(2-\rho)}{\Gamma\left(\alpha\left(n_{I I}-1\right)+2-\rho\right)} .
$$


Similarly, let $\bar{q}$ be defined as the probability of entry for which seller $\ell \in\{1,2\}$ is indifferent between selecting the FPA and the SPA given that seller $\ell^{\prime}$ offers an SPA.

$$
\sum_{n_{I}=0}^{N}\left(\begin{array}{c}
N \\
n_{I}
\end{array}\right)(\bar{q})^{n_{I}}(1-\bar{q})^{N-n_{I}} R_{I}\left(n_{I}, r\right)=0.5^{N} \sum_{n_{I I}=0}^{N}\left(\begin{array}{c}
N \\
n_{I I}
\end{array}\right) R_{I I}\left(n_{I I}\right)
$$

Note that because $R_{I}\left(n_{I}, r\right)>R_{I I}\left(n_{I I}\right)$ for any $n_{I}=n_{I I}$ and $r>0$, and because $E\left[R_{I}\right]$ is continuous and strictly increasing in $q$ (see proof of Lemma 4), the LHS of (10) will be larger than the RHS for any $q \geq 0.5$. Likewise, because $E\left[R_{I I}\right]$ is continuous and strictly decreasing in $q$ (see proof of Lemma 4), the LHS of (9) will be larger than the RHS for any $q \geq 0.5$. Therefore, both $q$ and $\bar{q}$ will be strictly below 0.5 .

Figure 1 illustrates the values of $q^{*}, q$ and $\bar{q}$ for different values of $\alpha$ and $\rho$ and for $N=4$. The numbered regions in Fig. 1 correspond to different equilibrium outcomes.

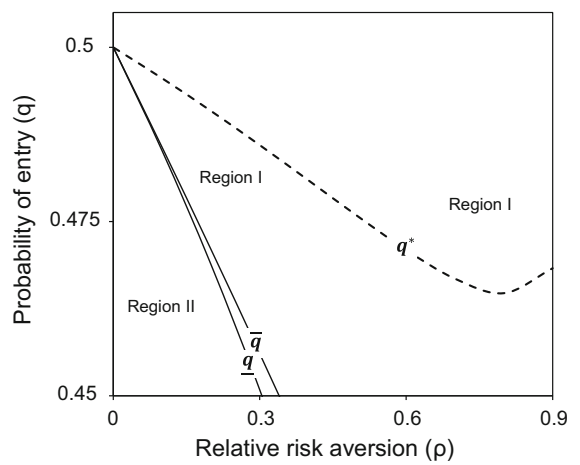

(a)

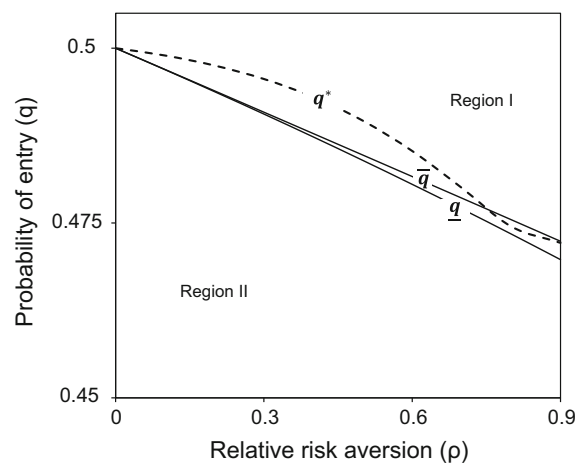

(c)

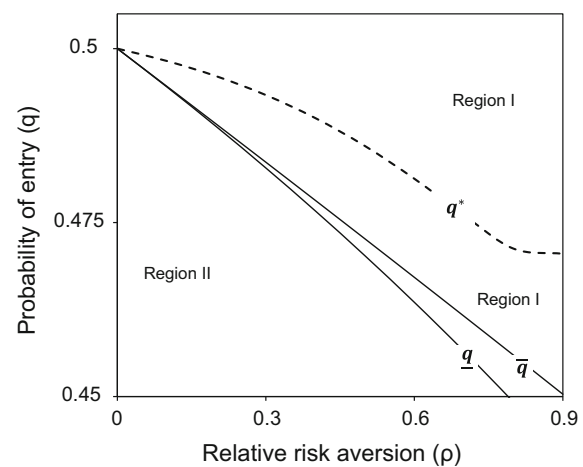

(b)

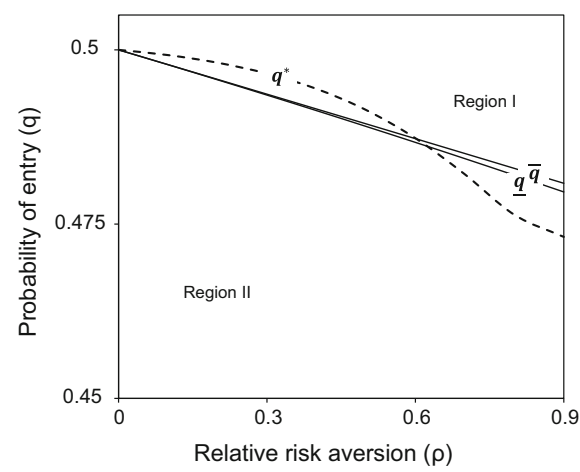

(d)

Fig. 1 Effect of the distribution of values on auction selection with CRRA bidders (where $F=v^{\alpha}$ and $N=4)$. a $\alpha=1$. $\mathbf{b} \alpha=5$. $\mathbf{c} \alpha=10$. $\mathbf{d} \alpha=15$ 
In region $\mathrm{I}$, where $q>q, \bar{q}$, sellers have a dominant strategy to select the FPA. As a result, in this region there is a unique Nash equilibrium and it is given by the strategy profile (FPA, FPA). In region II, where $q<q, \bar{q}$, the unique Nash equilibrium is given by (SPA, SPA). In region III (visible for some parameter values in Fig. 1, but not explicitly indicated), it is the case that $q<q<\bar{q}$. As $E\left[R_{I}\right]$ is increasing in $q$ and $E\left[R_{I I}\right]$ is decreasing in $q$, it follows that in this case, the auction selection game is in fact a coordination game. The Nash equilibria are then given by (FPA, FPA), (SPA, SPA) and one involving mixed strategies. ${ }^{12}$

Figure 1a shows that, when values are uniformly distributed, $q^{*}$ remains above $q$ and $\bar{q}$ for any $\rho \in(0,1)$. This implies that sellers have a dominant strategy to select the FPA. However, as the distribution function becomes more skewed ( $\alpha$ becomes larger), $q$ and $\bar{q}$ shift upwards, leading to an increase in region II at the expense of region I. As a result, we find that if the distribution of values is sufficiently skewed and bidders are sufficiently risk averse, then $q^{*}$ also moves through regions II and III (see Fig. 1c, d), such that in equilibrium both sellers could also end up selecting SPAs. Our finding is analogous to that of Smith and Levin (1996), who show, in a model where bidders can choose whether or not to enter an auction at an entry cost, that the traditional revenue ranking for risk averse bidders can be reversed if the distribution of values is sufficiently skewed. The reason for these results is that an increase in $\alpha$ reduces the variance in payments generated in the SPA and therefore decreases the difference in ex ante expected revenues between the FPA and SPA. This can immediately be seen from (5) and (6), where an increase in $\alpha$ leads to a relatively larger change in the ex ante expected revenue for the SPA than for the FPA.

Smith and Levin (1996) suspect that increasing the number of bidders $(N)$ affects the revenue ranking between the FPA and SPA in a similar way as increasing the skewness of the distribution does $(\alpha)$. They therefore "conjecture that SPA would tend to be favored by the seller more often in markets with many potential bidders than in markets with few" (Smith and Levin 1996, p. 558). We find that this does not hold for our setting. Rather, we find that increasing the number of bidders decreases both $q$ and $\bar{q}$, thereby making it less likely that the dominance of FPA is overthrown. Figures 2 and 3 show the effect of increasing $N$ to 6 and 9 when values are uniformly distributed $(\alpha=1)$ and when the distribution of values is rather skewed $(\alpha=15)$, respectively. This finding extends to larger $N$ as well.

\footnotetext{
12 Note that there may exist a fourth possible equilibrium outcome, i.e., where $q>q>\bar{q}$. In this case, the auction selection game is in fact an anti-coordination game, such that the resulting Nash equilibria are given by (FPA, SPA), (SPA, FPA) and an equilibrium involving mixed strategies. While we do not find any evidence for cases where $q>\bar{q}$ in our simulations, we cannot rule out that such cases exist for certain distribution functions or utility functions.
} 


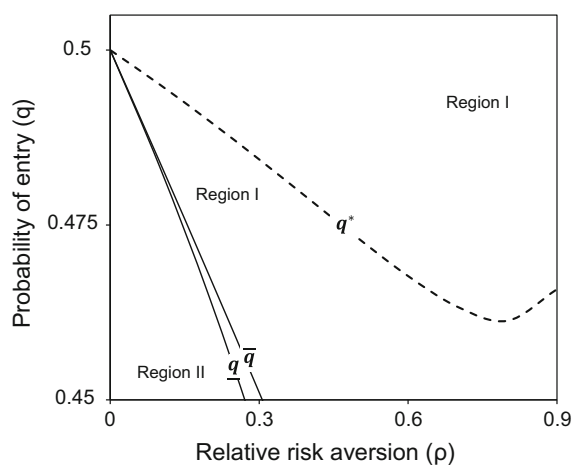

(a)

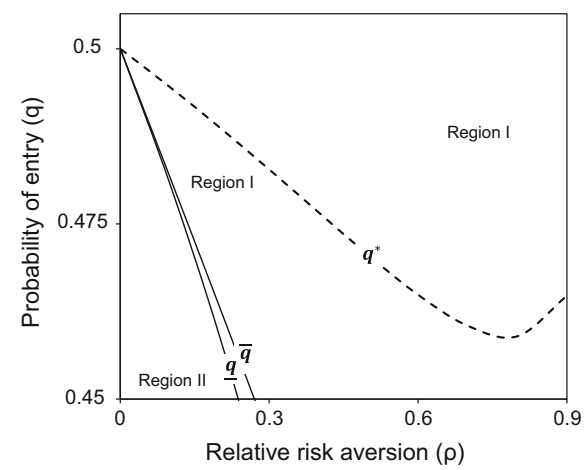

(b)

Fig. 2 Effect of $N$ on auction selection with CRRA bidders and a uniform distribution of values (where $F=v^{\alpha}$ and $\left.\alpha=1\right)$. $\mathbf{a} N=6 . \mathbf{b} N=9$

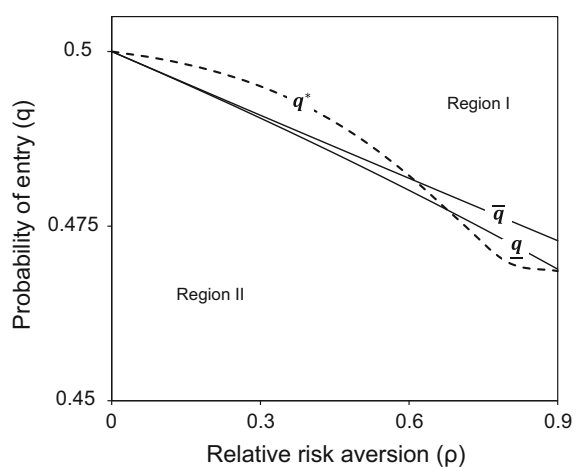

(a)

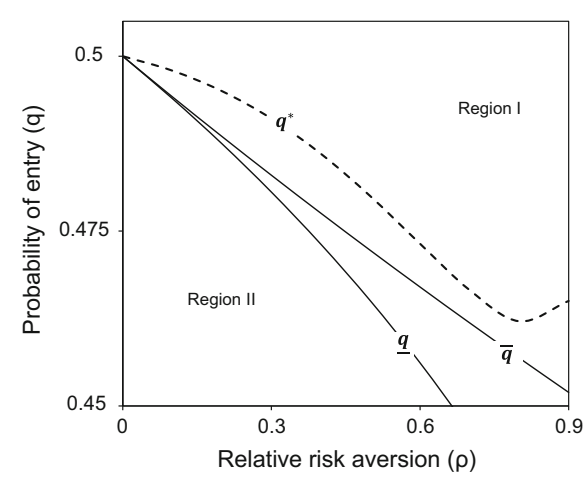

(b)

Fig. 3 Effect of $N$ on auction selection with CRRA bidders and a skewed distribution of values (where $F=v^{\alpha}$ and $\left.\alpha=15\right)$. $\mathbf{a} N=6 . \mathbf{b} N=9$

\section{Bidder heterogeneity}

We now consider to what extent our results are maintained when bidders are heterogeneous in their risk preferences (Sect. 5.1) or heterogeneous in their values (Sect. 5.2), meaning that bidders know their independent and private values before entering auctions. Such extensions are important, as in the symmetric entry equilibria described in Proposition 1 bidders mix between the auctions with specific probabilities, in spite of being indifferent between them. With bidder heterogeneity, these mixed equilibria are reinterpreted as instances where some bidder types prefer FPAs and other bidder types prefer SPAs.

\subsection{Heterogeneous risk preferences}

To examine how introducing heterogeneity in risk aversion affects our results, let us assume that each bidder's absolute rate of risk aversion is given by $r=\gamma+\delta v$, where 
$v$ denotes the bidder's value, and $\gamma$ and $\delta$ are parameters defining the bidder's type. We consider two cases of heterogeneity. In Case 1, given that we have previously looked separately at bidders with CARA, DARA and IARA utility functions, we assume that all those bidder types are present in the market. That is, every bidder has the same $\gamma>0$, but bidders differ according to $\delta$, which is independently distributed over $[\underline{\delta}, \bar{\delta}]$, with $\underline{\delta}<0$ and $\bar{\delta}>0$, and follows a cumulative distribution function $G^{1}$. This means that bidders with $\underline{\delta}<\delta<0$ exhibit DARA, bidders with exactly $\delta=0$ exhibit CARA, and bidders with $0<\delta<\bar{\delta}$ exhibit IARA. In Case 2, all bidders have the same $\delta$, but differ according to $\gamma$, which is independently distributed over $[\gamma, \bar{\gamma}]$ and follows a cumulative distribution function $G^{2}$, such that bidders may be more or less risk averse overall.

Denote generically by $\varepsilon$ the parameter according to which bidders differ, with $\varepsilon=\delta$ in Case 1 and $\varepsilon=\gamma$ in Case 2. The game described in Sect. 2 is then modified such that at the beginning of Stage 2, prior to deciding which auction to enter, each bidder additionally finds out her own type $\varepsilon$. The distribution of types, $G^{k}(\varepsilon)$ with $k \in\{1,2\}$ referring to either Case 1 or 2 , is common knowledge. The rest of the game proceeds as before.

To analyze the robustness of our results, we start by considering bidders' entry decisions in each Stage 2 subgame. Let us first point out that Lemma 1, which states that equal auction formats are entered with equal probability, continues to apply. Hence, like before, we need only consider bidders' entry decisions between one of the FPAs and one of the SPAs. In accordance with Pevnitskaya (2004) and Palfrey and Pevnitskaya (2008), we conjecture that any symmetric entry equilibrium with heterogenously risk averse bidders is characterized by a cutoff value $\varepsilon^{*}$, where bidders with $\varepsilon<\varepsilon^{*}$ self-select into the SPA, and bidders with $\varepsilon>\varepsilon^{*}$ self-select into the FPA. Such a symmetric entry equilibrium is implicitly defined by the following equality for $\varepsilon=\varepsilon^{*}$ :

$$
\begin{gathered}
\sum_{n_{I}=1}^{N}\left(\begin{array}{c}
N-1 \\
n_{I}-1
\end{array}\right)\left(\frac{1-G^{k}\left(\varepsilon^{*}\right)}{m_{I}}\right)^{n_{I}-1}\left(1-\frac{1-G^{k}\left(\varepsilon^{*}\right)}{m_{I}}\right)^{N-n_{I}} E\left[u \mid I, n_{I}, \varepsilon\right] \\
=\sum_{n_{I}=1}^{N}\left(\begin{array}{c}
N-1 \\
n_{I I}-1
\end{array}\right)\left(\frac{G^{k}\left(\varepsilon^{*}\right)}{m_{I I}}\right)^{n_{I I}-1}\left(1-\frac{G^{k}\left(\varepsilon^{*}\right)}{m_{I I}}\right)^{N-n_{I I}} E\left[u \mid I I, n_{I I}, \varepsilon\right]
\end{gathered}
$$

where $E\left[u \mid I, n_{I}, \varepsilon\right]$ (respectively, $E\left[u \mid I I, n_{I I}, \varepsilon\right]$ ) is the ex ante expected utility of a bidder of type $\varepsilon$ in an FPA (respectively, an SPA) when finding out that $n_{I}-1$ (respectively, $n_{I I}-1$ ) competing bidders have entered. The line of reasoning in Proposition 1 suggests that $1-G^{k}\left(\varepsilon^{*}\right)=\left(m_{I} / M\right)$ if the cutoff bidder exhibits CARA, $1-G^{k}\left(\varepsilon^{*}\right)<\left(m_{I} / M\right)$ if the cutoff bidder exhibits DARA, and $1-G^{k}\left(\varepsilon^{*}\right)>\left(m_{I} / M\right)$ if the cutoff bidder exhibits IARA.

Let us now consider how (11) is affected when marginally changing the bidder's type $\varepsilon$. Applying the envelope theorem, we only need to consider the direct effects of marginally increasing $\varepsilon$ on the ex ante expected utilities in the auctions and can thus ignore any indirect effects this may have through the bidding function. In both cases of heterogeneous risk preferences considered, an increase in $\varepsilon$ implies an increase in risk aversion. In Case 2, this is obvious; in Case 1, increasing $\delta$ weakly increases each 
bidder's $r$ for any utility function. For a systematic discussion, we regard situations in which the cutoff bidder exhibits CARA, DARA and IARA separately.

In any symmetric entry equilibrium where the cutoff bidder exhibits CARA, $1-G^{k}\left(\varepsilon^{*}\right)=\left(m_{I} / M\right)$ implies that bidders face the same distribution over the number of competing bidders in each auction format. Moreover, for any given number of competing bidders, for each $v$, bidders have the same probability of winning in each of the auction formats. The lottery corresponding to entering the SPA in the RHS of (11) therefore only differs from the lottery corresponding to entering the FPA in the LHS, in that a winning bidder in the SPA faces a distribution of prices that she could pay rather than a single price. The risk averse cutoff bidder $\varepsilon^{*}$ is only indifferent between entering the SPA and the FPA because overbidding occurs in the latter. Let us now marginally increase $\varepsilon$ above $\varepsilon^{*}$. Then, as the SPA is riskier, an increase in $\varepsilon$ means that the FPA is preferred. The opposite is true if $\varepsilon$ is decreased.

Next, in any symmetric entry equilibrium where the cutoff bidder exhibits DARA, $1-G^{k}\left(\varepsilon^{*}\right)<\left(m_{I} / M\right)$ indicates that the distribution over the number of bidders is skewed toward larger numbers of bidders in the SPA and skewed toward smaller numbers of bidders in the FPA. It continues to be the case that, for any given number of bidders, a bidder faces a riskier lottery in the SPA than in the FPA. If we marginally increase $\varepsilon$ above $\varepsilon^{*}$, the bidder will thus prefer the FPA, both because it is less risky, and because it continues to be skewed toward smaller numbers of bidders. Conversely, in any symmetric entry equilibrium where the cutoff bidder exhibits IARA, $1-G^{k}\left(\varepsilon^{*}\right)>$ $\left(m_{I} / M\right)$ indicates that the distribution over the number of bidders is skewed toward smaller numbers of bidders in the SPA and skewed toward larger numbers of bidders in the FPA. Now, if we marginally decrease a bidder's $\varepsilon$ below $\varepsilon^{*}$, the bidder will prefer the SPA as she cares less about risk than the cutoff bidder, and because the SPA continues to be skewed toward smaller numbers of bidders. This suggests that whether we consider Case 1 or Case 2, and whether the cutoff bidder exhibits CARA, DARA or IARA, in any symmetric entry equilibrium bidders with $\varepsilon<\varepsilon^{*}$ enter the SPA, whereas bidders with $\varepsilon>\varepsilon^{*}$ enter the FPA.

Our results for each Stage 2 subgame for homogeneous risk preferences in Proposition 1 can be reinterpreted as approximating the results for heterogeneous risk preferences. Applying Harsanyi's (1973) purification theorem, consider a perturbed version of each original Stage 2 subgame, where rather than having a single bidder type with one specific risk aversion parameter, each bidder's parameter is independently chosen from the same density function over a continuum of parameters, distributed around the original parameter. Each bidder knows her own risk aversion parameter, but does not know the parameters of the other bidders, though the distribution over parameters is common knowledge. We then obtain an incomplete information game as described above, in which every bidder type plays a pure strategy, but where from the perspective of the individual bidder, other bidders continue to choose their strategies probabilistically. The purification theorem now states that starting from the pure-strategy equilibrium of such an incomplete information game, and taking the limit such that the continuum of parameters shrinks to the original parameter, one approaches the original mixed-strategy equilibrium. The mixed-strategy equilibrium itself can now be interpreted as the limit case of a pure-strategy equilibrium where one range of bidder types chooses one, and the other range of bidder types chooses 
the other strategy. This in turn suggests that the results in Proposition 2 for Stage 1, where sellers' auction selection decisions are considered, are maintained as long as the distribution of risk aversion parameters lies close to the originally considered unique parameter. We conclude that our results are robust to a small degree of heterogeneity in bidders' risk preferences.

We finally confront our predictions for bidders with heterogeneous risk aversion with experimental findings. In an experimental study, where bidders choose between entering an English auction and an FPA, Ivanova-Stenzel and Salmon (2008a) find no support for self-selection based on risk aversion. To analyze the self-selection effect, the authors use the amount of overbidding as a measure of risk aversion. More specifically, they assume that bidders' preferences can be described by $u\left(x_{i}\right)=x_{i}^{1-\rho}$, which results in an equilibrium bidding strategy $b\left(v \mid I, n_{I}\right)=\left(\left(n_{I}-1\right) /\left(n_{I}-\rho\right)\right) v$ when values are uniformly distributed between $[0,1]$. They find that neither risk aversion nor, more generally, the degree of overbidding has a statistically significant effect on bidders' entry decisions. At first sight, this does not seem to be in line with our findings. It would therefore be interesting to investigate what drives the differences between our predictions and these experimental results.

\subsection{Known values}

The assumption that bidders do not know their own value before entering an auction is motivated by examples where bidders may only learn their exact value for the good for sale once they actually participate in the auction, e.g., for goods that come in many different varieties and require some research. Pevnitskaya (2004) gives an example of antique auctions, where sellers often advertise general inventory and where bidders can determine their exact value only after coming to the auction house and examining the goods prior to sale. As a result, these bidders do know the distribution of values, but only know their independent private value after entering the auction. In online auctions, this may occur in particular for experience goods such as leisure activities or holidays, where a single purchase may include many different options. Alternatively, there may be instances where assuming that bidders know their own value before entering an auction is more suitable. For example, this may occur in auctions for collector's items, where experienced collectors have a precise idea of the value they attach to a specific object before entering any of the auctions in which such an object is offered. We now examine how our results are affected by allowing bidders to find out their own value before deciding which auction to enter, where we continue to assume that each bidder has the same utility function. More specifically, the game described in Sect. 2 is now modified such that each bidder learns her own value $v$ at the beginning of Stage 2, prior to deciding which auction to enter.

Let us start by examining bidders' entry decisions within each Stage 2 subgame. Following Menezes and Monteiro (2000), we first consider whether any symmetric entry equilibrium can be characterized by a cutoff value, such that bidders with a value above this cutoff enter one auction format, and bidders with a value below the cutoff enter another auction format. An immediate problem with such an equilibrium seems to be that any bidder with a value above the cutoff has an incentive to enter the auction 
chosen by bidders with values below the cutoff, as this will ensure that she wins the auction and pays a lower price than she currently would. ${ }^{13}$

This suggests that when bidders know their values before entry, in any symmetric entry equilibrium of a Stage 2 subgame each bidder continues to mix over the available auctions. Indeed, consider the case where all bidders have the same CARA utility function. Now consider a focal bidder, while assuming that all competing bidders choose between auctions as described in Proposition 1. As Matthews's (1987) results hold regardless of whether bidders know their values or not, it follows that each bidder has a weak best response to choose each auction with the same probability, regardless of her value.

Now consider the case where all bidders have the same DARA utility function. Again, consider a focal bidder, while assuming that all competing bidders follow the strategy in Proposition 1, such that, regardless of their values, they enter any SPA with the same, higher, probability than any FPA. Given that the focal bidder is indifferent between the FPA and the SPA if she does not know her value, and given that the range of values is a continuum, there must be some intermediate value $v^{*}$ for which the bidder who does know her value is also indifferent. Now, marginally decrease the bidder's value below $v^{*}$. By DARA, this implies that the bidder becomes more risk averse. Following the same reasoning as in Sect. 5.1, she will then prefer the FPA over the SPA. This shows that, unlike the case of CARA, with a DARA utility function a symmetric entry equilibrium where all bidders mix with the same probability, independently of their value, does not exist. This suggests that in any mixed equilibrium, bidders should mix with a different probability depending on their value. We conjecture that, if bidders exhibit DARA, bidders with higher values, who are less risk averse, will enter the SPA more frequently, whereas bidders with lower values, who are more risk averse, will enter the FPA more frequently. By the same reasoning, the opposite would be true for bidders that exhibit IARA.

Following the customary assumption that DARA utility functions are empirically more plausible, our prediction could explain experimental findings by Ivanova-Stenzel and Salmon (2011), who find that bidders with low values choose the FPA more often, while bidders with high values choose the ascending auction more often. Yet, contradicting such an explanation, these authors also again find that bidders' entry decisions are not affected by their degree of risk aversion, as measured by the amount of overbidding in the FPA. Again, the question on the driving factors of this difference between our predictions and the mentioned experimental results arises.

We conclude by looking at the implications for sellers' auction selection decisions in Stage 1 of our game. Given our conjectures about entry decisions when bidders know their own value in each Stage 2 subgame, our results in Proposition 2 seem to be maintained. If bidders exhibit CARA and continue to follow the strategy described

\footnotetext{
13 An exception may be a case where very few bidders enter the auction chosen by a range of high-value bidders. If it is sufficiently likely to find oneself alone in the auction such that one can bid a minimal price, high-value bidders may still prefer this auction. However, it cannot be excluded that low-value bidders would prefer this auction for the same reason. Also, the simulations in Sect. 4.1 suggest that in the mixed equilibria in Proposition 1 auctions that are entered more frequently are only entered marginally more often. Following Harsanyi's (1973) purification theorem (see Sect. 5.1), considering these mixed equilibria as limit cases of incomplete information games, it should continue to be the case that no auction is chosen substantially more often than any other auction.
} 
in Proposition 1, sellers will continue to have a dominant strategy to select the FPA. If bidders exhibit IARA, it continues to be the case that FPAs are entered more frequently overall. Moreover, FPAs may be entered more often by high-value bidders. This strengthens our results in Proposition 2. Finally, if bidders exhibit DARA, SPAs are entered more frequently overall, and may also be entered more often by high-value bidders. However, as risk averse bidders continue to overbid in the FPA, the results remain indeterminate.

\section{Affiliated private values}

In the previous, we have assumed that bidders have independent private values (IPV) and showed that, unless bidders have DARA utility functions, sellers prefer to select FPAs. In Sect. 4.1, we demonstrated that if bidders exhibit DARA, sellers may have an incentive to select SPAs as well. This is most likely to happen when the distribution of values is skewed toward higher values, which may be interpreted as inducing stronger competition between bidders. We therefore now consider another source of increasing competition between bidders: affiliation. This can be interpreted as positive correlation, where values are jointly distributed in such a way that the individual bidder with a high value expects the distribution of other bidders to be skewed toward higher values (see Milgrom and Weber 1982 for a precise definition). As an example, Kagel et al. (1987, p. 1275) refer to "charity fundraisers of consumer perishables, where an item unusually appealing to you is typically more appealing to other bidders as well". In this section, we therefore consider the implications of relaxing the assumption of IPV, instead assuming that bidders have affiliated private values (APV).

To examine the implications of allowing for APV, let us first consider how this affects each Stage 2 subgame, where bidders decide which auction format to enter. Note that with APV the utility equivalence for risk neutral and CARA bidders no longer holds. Milgrom and Weber (1982) find that, if a fixed number of risk neutral bidders have APV, the expected payment in the SPA is greater than in the FPA. As a result, risk neutral bidders prefer the FPA to the SPA. This follows from the linkage principle, which implies that the auction formats that reveal affiliated information in the course of the auction, or use it in determining the price, will result in higher prices. As the latter happens in the SPA, while no information is revealed in the FPA, the expected payment in the SPA will be greater than in the FPA. This follows from the fact that with an FPA, for any given bid that an individual bidder may make, conditional on winning, increasing her value does not increase her expected payment, simply because she pays her bid. With an SPA, for any given bid that an individual bidder may make, conditional on winning, increasing her value does increase her expected payment, because she expects others to have higher values. Matthews (1987) argues that the linkage principle results in a preference for the FPA if APV bidders exhibit CARA or IARA. If APV bidders exhibit DARA, however, which auction format is preferred depends on how sharp the absolute rate of risk aversion decreases as a function of the value relative to how strong affiliation is.

As risk neutral, CARA and IARA APV bidders prefer the FPA to the SPA, more bidders will enter the FPA in equilibrium (i.e., $\left.q^{*}>\left(m_{I} / M\right)\right)$. Hence, whereas Propo- 
sition 1(iii) is robust to allowing for APV, Lemma 3 and Proposition 1(i), which both predict that FPAs and SPAs will be entered with equal probability, are changed even when we allow for the slightest bit of affiliation. DARA bidders will prefer the SPA if the rate at which the absolute rate of risk aversion decreases as a function of the value is high; they will prefer the FPA if affiliation is strong. This suggests that Proposition 1(ii), which predicts that bidders are more likely to enter SPAs, is maintained as long as the absolute rate of risk aversion decreases strongly as a function of value, and as long as affiliation is weak.

We now turn to Stage 1 of our game, where sellers select which auction format they offer. Recall that, with risk neutral APV bidders, the ex ante expected revenue of the SPA is greater than that of the FPA (e.g., Milgrom and Weber 1982). Combining this with our result for risk neutral APV bidders in each Stage 2 subgame produces an uncertain outcome of the auction selection game: whereas the higher number of bidders in the FPA leads competing sellers to prefer the FPA, the higher expected payment for a fixed number of bidders in the SPA leads these sellers to prefer the SPA.

The ranking of ex ante expected revenues is less clear-cut when APV bidders are risk averse. Whereas affiliation leads the SPA to dominate the FPA in terms of ex ante expected revenues, risk aversion has the opposite effect. Which effect predominates thus depends on the strength of affiliation versus the strength of risk aversion. In a series of laboratory experiments, Kagel et al. (1987) indeed find that the FPA generates more revenue than the SPA when bidders' values are weakly affiliated, whereas the opposite ranking is found when bidders' values are strongly affiliated. This suggests the following for the robustness of Proposition 2.

With CARA and IARA APV bidders, by the above, if affiliation is comparatively weak, all sellers will have a dominant strategy to select the FPA and Proposition 2 is maintained. If affiliation is comparatively strong, then for a fixed number of bidders, sellers prefer the SPA and bidders prefer the FPA. In this case, we do not have a clear prediction. Hence, our results for Proposition 2 are robust to assuming a limited amount of affiliation. With DARA APV bidders, if risk aversion decreases at a strong rate, risk aversion will also be strong overall, meaning that affiliation is comparatively weak. In this case, similar to the scenario with DARA IPV bidders, bidders prefer the SPA, while sellers prefer the FPA. However, if affiliation is comparatively strong (meaning that risk aversion decreases at a relatively slow rate and risk aversion is also weak overall), then for a fixed number of bidders, sellers prefer the SPA and bidders prefer the FPA. As more bidders enter the FPA in equilibrium, sellers may still have an incentive to offer FPAs alongside SPAs. Finally, one could imagine that risk aversion decreases at a weak rate, but is at the same time very strong overall, such that affiliation is weak compared to overall risk aversion. In this case, sellers continue to prefer the FPA. If affiliation is still relatively strong compared to the rate at which risk aversion decreases, bidders also prefer the FPA. Hence, the FPA is offered. However, if affiliation is also weak compared to the rate at which risk aversion decreases, bidders prefer the SPA. In this case, our results are again robust to allowing for APV.

The prevalence of English auctions on the Internet suggests that APV may be a more realistic assumption in the context of online auctions. It would therefore to be interesting to investigate whether bidders' private values in online auctions are indeed affiliated instead of independent. 


\section{Conclusion}

The main objective of this paper is to investigate which auctions are selected by competing sellers when they may choose between first-price and second-price auctions and when risk averse bidders endogenously enter one of the auctions. We construct a three-stage game in which two units of a homogenous good are offered simultaneously to a group of $N$ homogeneously risk averse bidders. At Stage 1, the sellers each select an auction; at Stage 2, each bidder learns which auctions have been selected and decides to enter one of the auctions; finally, at Stage 3, the auctions are conducted.

Our key findings can be summarized along two lines. First, we show that when bidders may choose between entering first-price and second-price auctions, then a symmetric entry equilibrium exists involving mixed strategies, where the mixing probabilities depend on whether bidders exhibit constant, decreasing or increasing absolute risk aversion. If bidders exhibit risk neutrality or constant absolute risk aversion, they will enter each auction with equal probability. If bidders exhibit decreasing absolute risk aversion, however, they will enter second-price auctions with greater likelihood, and if bidders exhibit increasing absolute risk aversion, they will enter first-price auctions with greater likelihood. Second, we find that if bidders exhibit nondecreasing absolute risk aversion, competing sellers have a dominant strategy to select first-price auctions. We demonstrate by example that if bidders exhibit decreasing absolute risk aversion, sellers may also select second-price auctions if the distribution of private values is sufficiently skewed. Our results are robust to allowing for a limited degree of heterogeneity in risk aversion and affiliation, as well as allowing bidders to find out their own value before deciding which auction to enter.

Whereas traditional revenue ranking theorems predict that competing sellers should prefer the first-price auction when bidders are risk averse, in reality most sellers seem to offer English auctions, which are strategically equivalent to second-price auctions. Our analysis suggests that this could be explained by the presence of decreasing absolute risk aversion. Additionally, even though experimental studies often assume that values are uniformly distributed, it is possible that in many real-world auctions values actually follow a more skewed distribution. Future research might further explore this, both experimentally and empirically.

In the context of online auctions, it would also be interesting to explore to which extent our findings depend on the assumption that bidders know how many other bidders actually enter each auction. After all, on the Internet, bidders may not be aware of how many competing bidders participate in an auction. Matthews (1987) shows that the preference rankings for risk averse bidders can be extended to a setting where the number of bidders participating in each auction is concealed. We therefore conjecture that in such a setting, there exists an entry equilibrium analogous to the one we find in this paper. Future research may consider the effects of concealing the number of competing bidders on bidders' entry decisions and its implications for the auction selection decisions of competing sellers. 
Open Access This article is distributed under the terms of the Creative Commons Attribution 4.0 International License (http://creativecommons.org/licenses/by/4.0/), which permits unrestricted use, distribution, and reproduction in any medium, provided you give appropriate credit to the original author(s) and the source, provide a link to the Creative Commons license, and indicate if changes were made.

\section{Appendix 1: Polynomials in Bernstein form}

For any sequence $\left(c_{0}, c_{1}, \ldots, c_{n}\right)$ and for any $t \in[0,1]$, define the function $\sum_{k=0}^{n}\left(\begin{array}{l}n \\ k\end{array}\right)(1-t)^{n-k} t^{k} c_{k}$ as a polynomial in Bernstein form (Farouki 2012, p. 385). Both the bidders' ex ante expected utility of entering an auction (1) and the sellers' expected revenue of offering an auction (3)-(4) in our model have the form of such a polynomial. The property of polynomials in Bernstein form in Lemma 5 (Property 11, p. 391 in Farouki 2012) will be useful for several of our results. We show this property formally.

Lemma 5 For any $t \in[0,1]$ and for any sequence $\left(c_{0}, c_{1}, \ldots, c_{n}\right)$, the function $\varphi(t)=\sum_{k=0}^{n}\left(\begin{array}{l}n \\ k\end{array}\right)(1-t)^{n-k} t^{k} c_{k}$ is continuous in $t$. Furthermore, $\varphi(t)$ is monotonically increasing (decreasing) in $t$ if, for any $k \in[0, n-1], c_{k+1} \geq c_{k}\left(c_{k+1} \leq c_{k}\right)$. Similarly, $\varphi(t)$ is strictly increasing (decreasing) in $t$ if, for any $k \in[0, n-1]$, $c_{k+1}>c_{k}\left(c_{k+1}<c_{k}\right)$.

Proof This proof consists of two steps. First, we argue that $\varphi(t)$ is continuous in $t$. Second, we show that how $\varphi(t)$ changes in $t$ crucially depends on the difference between $c_{k+1}$ and $c_{k}$.

Step 1 Each term of $\varphi(t)$ consists of a binomial probability that is continuous in $t$, multiplied by a coefficient that is not a function of $t$. It follows that each term of $\varphi(t)$ in continuous in $t$. Continuity of $\varphi(t)$ then follows from the fact that the sum of $n$ continuous terms is continuous as well.

Step 2 Note that $\varphi(t)$ is a polynomial in Bernstein form. One of the properties of such polynomials is that its derivative with respect to $t$ can be written as follows (Farouki 2012, p. 391).

$$
\begin{aligned}
\varphi^{\prime}(t) & =\frac{\partial}{\partial t}\left(\sum_{k=0}^{n}\left(\begin{array}{l}
n \\
k
\end{array}\right)(1-t)^{n-k} t^{k} c_{k}\right) \\
& =n \sum_{k=0}^{n-1}\left(\begin{array}{c}
n-1 \\
k
\end{array}\right)(1-t)^{n-1-k} t^{k}\left(c_{k+1}-c_{k}\right)
\end{aligned}
$$

To prove this property, note that $\varphi^{\prime}(t)$ can be written as follows.

$$
\begin{aligned}
\varphi^{\prime}(t) & =\frac{\partial}{\partial t}\left(\sum_{k=0}^{n}\left(\begin{array}{l}
n \\
k
\end{array}\right)(1-t)^{n-k} t^{k} c_{k}\right) \\
& =-\sum_{k=0}^{n-1}\left(\begin{array}{l}
n \\
k
\end{array}\right)(n-k)(1-t)^{n-1-k} t^{k} c_{k}+\sum_{k=1}^{n}\left(\begin{array}{l}
n \\
k
\end{array}\right) k(1-t)^{n-k} t^{k-1} c_{k}
\end{aligned}
$$


Now note that $\left(\begin{array}{l}n \\ k\end{array}\right)(n-k)=n\left(\begin{array}{c}n-1 \\ k\end{array}\right)$, and that $\left(\begin{array}{l}n \\ k\end{array}\right) k=n\left(\begin{array}{l}n-1 \\ k-1\end{array}\right)$. It follows that we can rewrite the last expression as

$$
\begin{aligned}
\varphi^{\prime}(t) & =-n \sum_{k=0}^{n-1}\left(\begin{array}{c}
n-1 \\
k
\end{array}\right)(1-t)^{n-1-k} t^{k} c_{k}+n \sum_{k=1}^{n}\left(\begin{array}{l}
n-1 \\
k-1
\end{array}\right)(1-t)^{n-k} t^{k-1} c_{k} \\
& =-n \sum_{k=0}^{n-1}\left(\begin{array}{c}
n-1 \\
k
\end{array}\right)(1-t)^{n-1-k} t^{k} c_{k}+n \sum_{k=0}^{n-1}\left(\begin{array}{c}
n-1 \\
k
\end{array}\right)(1-t)^{n-1-k} t^{k} c_{k+1} \\
& =n \sum_{k=0}^{n-1}\left(\begin{array}{c}
n-1 \\
k
\end{array}\right)(1-t)^{n-1-k} t^{k}\left(c_{k+1}-c_{k}\right)
\end{aligned}
$$

It now follows that the sign of $\varphi^{\prime}(t)$ depends on the sign of $c_{k+1}-c_{k}$ for any $k \in[0, n-1]$. For any $t \in[0,1]$ and any $k \in[0, n-1], \varphi^{\prime}(t) \geq 0$ if $c_{k+1}-c_{k} \geq 0$, $\varphi^{\prime}(t) \leq 0$ if $c_{k+1}-c_{k} \leq 0, \varphi^{\prime}(t)>0$ if $c_{k+1}-c_{k}>0$, and $\varphi^{\prime}(t)<0$ if $c_{k+1}-c_{k}<0$. This concludes our proof.

\section{Appendix 2: Example with CRRA bidders}

Suppose that bidder $i$ has a CRRA utility function of the form $u\left(x_{i}\right)=x_{i}^{(1-\rho)}$, where $x_{i}$ represents $i$ 's payoff and $\rho \in[0,1)$ represents the constant degree of relative risk aversion. Further suppose that values are distributed according to $F=v^{\alpha}$ for $v \in[0,1]$, where $\alpha \geq 1$ and takes integer values only. From Smith and Levin (1996), we know that the symmetric equilibrium in the FPA is then given by the bidding strategy

$$
b\left(v \mid I, n_{I}\right)=\frac{\alpha\left(n_{I}-1\right)}{\alpha\left(n_{I}-1\right)+1-\rho} v
$$

The ex ante expected revenue of the FPA is given by

$$
\begin{aligned}
R_{I}\left(n_{I}, r\right) & =\int_{0}^{1} n_{I}\left(\frac{\alpha\left(n_{I}-1\right)}{\alpha\left(n_{I}-1\right)+1-\rho} v\right) \alpha v^{\alpha-1} v^{\alpha\left(n_{I}-1\right)} \mathrm{d} v \\
& =\alpha n_{I} \frac{\alpha\left(n_{I}-1\right)}{\alpha\left(n_{I}-1\right)+1-\rho} \int_{0}^{1} v^{\alpha n_{I}} \mathrm{~d} v \\
& =\alpha n_{I} \frac{\alpha\left(n_{I}-1\right)}{\alpha\left(n_{I}-1\right)+1-\rho}\left[\frac{1}{\alpha n_{I}+1} v^{\alpha n_{I}+1}\right]_{0}^{1} \\
& =\frac{\alpha\left(n_{I}-1\right)}{\alpha\left(n_{I}-1\right)+1-\rho} \frac{\alpha n_{I}}{\alpha n_{I}+1}
\end{aligned}
$$

Given that there are $n_{I}$ bidders in the auction, each bidder then has an ex ante expected utility of

$$
E\left[u \mid I, n_{I}\right]=\int_{0}^{1} \alpha v^{\alpha-1} v^{\alpha\left(n_{I}-1\right)}\left(v-\frac{\alpha\left(n_{I}-1\right)}{\alpha\left(n_{I}-1\right)+1-\rho} v\right)^{1-\rho} \mathrm{d} v
$$




$$
\begin{aligned}
& =\alpha\left(\frac{1-\rho}{\alpha\left(n_{I}-1\right)+1-\rho}\right)^{1-\rho} \int_{0}^{1} v^{\alpha n_{I}-\rho} \mathrm{d} v \\
& =\alpha\left(\frac{1-\rho}{\alpha\left(n_{I}-1\right)+1-\rho}\right)^{1-\rho}\left[\frac{1}{\alpha n_{I}+1-\rho} v^{\alpha n_{I}+1-\rho}\right]_{0}^{1} \\
& =\frac{\alpha}{\alpha n_{I}+1-\rho}\left(\frac{1-\rho}{\alpha\left(n_{I}-1\right)+1-\rho}\right)^{1-\rho}
\end{aligned}
$$

For the SPA, the symmetric equilibrium is to bid one's own value, that is, $b\left(v \mid I I, n_{I I}\right)=v$. The ex ante expected revenue of the SPA is then given by

$$
\begin{aligned}
R_{I I}\left(n_{I I}\right) & =\int_{0}^{1} n_{I I}\left(n_{I I}-1\right) v \alpha v^{\alpha-1} v^{\alpha\left(n_{I I}-2\right)}\left[1-v^{\alpha}\right] \mathrm{d} v \\
& =\alpha n_{I I}\left(n_{I I}-1\right) \int_{0}^{1} v^{\alpha\left(n_{I I}-1\right)}\left(1-v^{\alpha}\right) \mathrm{d} v \\
& =\alpha n_{I I}\left(n_{I I}-1\right)\left\{\left[\frac{1}{\alpha\left(n_{I I}-1\right)+1} v^{\alpha\left(n_{I I}-1\right)+1}\right]_{0}^{1}-\left[\frac{1}{\alpha n_{I I}+1} v^{\alpha n_{I I}+1}\right]_{0}^{1}\right\} \\
& =\frac{\alpha\left(n_{I I}-1\right)}{\alpha\left(n_{I I}-1\right)+1} \frac{\alpha n_{I I}}{\alpha n_{I I}+1}
\end{aligned}
$$

Following Smith and Levin (1996), we show that, given that there are $n_{I I}$ bidders in the auction, each bidder has an ex ante expected utility of

$$
\begin{aligned}
E\left[u \mid I I, n_{I I}\right] & =\int_{0}^{1}\left[\alpha\left(n_{I I}-1\right) \int_{0}^{v} t^{\alpha\left(n_{I I}-1\right)-1}(v-t)^{1-\rho} \mathrm{d} t\right] \alpha v^{\alpha-1} \mathrm{~d} v \\
& =\frac{\alpha}{\alpha n_{I I}+1-\rho} \frac{\left(\alpha\left(n_{I I}-1\right)\right) !}{\left(\alpha\left(n_{I I}-1\right)+1-\rho\right) !}
\end{aligned}
$$

where $\left(\alpha\left(n_{I I}-1\right)+1-\rho\right) ! \equiv \prod_{i=1}^{\alpha\left(n_{I I}-1\right)}(i+1-\rho)$. To establish (12), we start by proving that the term in square brackets in (12), which represents the expected utility of a bidder with value $v$, can be rewritten as follows.

$$
\alpha\left(n_{I I}-1\right) \int_{0}^{v} t^{\alpha\left(n_{I I}-1\right)-1}(v-t)^{1-\rho} \mathrm{d} t=\frac{\left(\alpha\left(n_{I I}-1\right)\right) !}{\left(\alpha\left(n_{I I}-1\right)+1-\rho\right) !} v^{\alpha\left(n_{I I}-1\right)+1-\rho}
$$

Suppose that $\alpha\left(n_{I I}-1\right)=1$. Then (13) is trivially true.

$$
\int_{0}^{v}(v-t)^{1-\rho} \mathrm{d} t=-\frac{1}{2-\rho}\left[t^{2-\rho}\right]_{0}^{v}=\frac{1}{2-\rho} v^{2-\rho}
$$

Let us now show that (13) also holds for $\alpha\left(n_{I I}-1\right)=2$. In order to do so, we need to use integration by parts: $\int u \mathrm{~d} v=u v-\int v \mathrm{~d} u$. Integrating the LHS of (13) by parts gives us the following. 


$$
\begin{aligned}
& -\alpha\left(n_{I I}-1\right)\left[\frac{1}{2-\rho} t^{\alpha\left(n_{I I}-1\right)-1}(v-t)^{2-\rho}\right]_{0}^{v} \\
& +\alpha\left(n_{I I}-1\right) \frac{\alpha\left(n_{I I}-1\right)-1}{2-\rho} \int_{0}^{v} t^{\alpha\left(n_{I I}-1\right)-2}(v-t)^{2-\rho} \mathrm{d} t \\
& =\frac{\alpha\left(n_{I I}-1\right)\left(\alpha\left(n_{I I}-1\right)-1\right)}{2-\rho} \int_{0}^{v} t^{\alpha\left(n_{I I}-1\right)-2}(v-t)^{2-\rho} \mathrm{d} t
\end{aligned}
$$

Now suppose that $\alpha\left(n_{I I}-1\right)=2$. The RHS of (14) then becomes

$$
\begin{aligned}
& \frac{\alpha\left(n_{I I}-1\right)\left(\alpha\left(n_{I I}-1\right)-1\right)}{2-\rho} \int_{0}^{v}(v-t)^{2-\rho} \mathrm{d} t \\
& =-\frac{\alpha\left(n_{I I}-1\right)\left(\alpha\left(n_{I I}-1\right)-1\right)}{2-\rho}\left[\frac{1}{3-\rho}(v-t)^{3-\rho}\right]_{0}^{v} \\
& =\frac{2 * 1}{(2-\rho)(3-\rho)} v^{3-\rho}
\end{aligned}
$$

which proves that (13) holds for $\alpha\left(n_{I I}-1\right)=2$ as well. Having verified (13) for $\alpha\left(n_{I I}-1\right)=\{1,2\}$ we now prove by induction. Assume that (13) holds for $\alpha\left(n_{I I}-1\right)=k$.

$$
k \int_{0}^{v} t^{k-1}(v-t)^{1-\rho} \mathrm{d} t=\frac{k !}{(k+1-\rho) !} v^{k+1-\rho}
$$

Now, we can show that (13) also holds for $\alpha\left(n_{I I}-1\right)=k+1$. That is, we want to prove the following.

$$
(k+1) \int_{0}^{v} t^{k}(v-t)^{1-\rho} \mathrm{d} t=\frac{(k+1) !}{((k+1)+1-\rho) !} v^{(k+1)+1-\rho}
$$

We start by integrating the LHS of (16). This gives us the following.

$$
\begin{aligned}
(k & +1)\left\{-\left[\frac{1}{2-\rho} t^{k}(v-t)^{2-\rho}\right]_{0}^{v}+\frac{k}{2-\rho} \int_{0}^{v} t^{k-1}(v-t)^{2-\rho} \mathrm{d} t\right\} \\
& =\frac{(k+1)}{(2-\rho)}\left\{k \int_{0}^{v} t^{k-1}(v-t)^{2-\rho} \mathrm{d} t\right\}
\end{aligned}
$$

We now use (15) to rewrite this as follows.

$$
\frac{(k+1)}{(2-\rho)}\left\{\frac{k !}{(k+2-\rho) !} v^{k+2-\rho}\right\}=\frac{(k+1) !}{(k+2-\rho) !} v^{k+2-\rho}
$$

This establishes (16) and concludes the proof of (13). Therefore, we can write the ex ante expected utility, where the bidder does not know her private value yet, as follows.

$$
E\left[u \mid I I, n_{I I}\right]=\int_{0}^{1}\left[\frac{\left(\alpha\left(n_{I I}-1\right)\right) !}{\left(\alpha\left(n_{I I}-1\right)+1-\rho\right) !} v^{\alpha\left(n_{I I}-1\right)+1-\rho}\right] \alpha v^{\alpha-1} \mathrm{~d} v
$$




$$
\begin{aligned}
& =\alpha \frac{\left(\alpha\left(n_{I I}-1\right)\right) !}{\left(\alpha\left(n_{I I}-1\right)+1-\rho\right) !} \int_{0}^{1} v^{\alpha n_{I I}-\rho} \mathrm{d} v \\
& =\alpha \frac{\left(\alpha\left(n_{I I}-1\right)\right) !}{\left(\alpha\left(n_{I I}-1\right)+1-\rho\right) !}\left[\frac{1}{\alpha n_{I I}+1-\rho} v^{\alpha n_{I I}+1-\rho}\right]_{0}^{1} \\
& =\frac{\alpha}{\alpha n_{I I}+1-\rho} \frac{\left(\alpha\left(n_{I I}-1\right)\right) !}{\left(\alpha\left(n_{I I}-1\right)+1-\rho\right) !}
\end{aligned}
$$

This concludes the proof of (12).

Notice that when bidders are risk neutral $(\rho=0)$ and when $n_{I}=n_{I I}$, then the FPA and SPA are both revenue and utility equivalent.

$$
\begin{aligned}
R_{I}\left(n_{I}, 0\right) & =\frac{\alpha\left(n_{I}-1\right)}{\alpha\left(n_{I}-1\right)+1-0} \frac{\alpha n_{I}}{\alpha n_{I}+1} \\
& =\frac{\alpha\left(n_{I I}-1\right)}{\alpha\left(n_{I I}-1\right)+1} \frac{\alpha n_{I I}}{\alpha n_{I I}+1}=R_{I I}\left(n_{I I}\right) \\
E\left[u \mid I, n_{I}\right] & =\frac{\alpha}{\alpha n_{I}+1-0}\left(\frac{1-0}{\alpha\left(n_{I}-1\right)+1-0}\right)^{1-0} \\
& =\frac{\left(\alpha\left(n_{I I}-1\right)\right) !}{\left.\left(\alpha\left(n_{I I}-1\right)+1-0\right)\right) !} \frac{\alpha}{\alpha n_{I I}+1-0}=E\left[u \mid I I, n_{I I}\right]
\end{aligned}
$$

\section{Appendix 3: Monopoly}

Recently, some sellers have started offering a single good in multiple selling mechanisms at the same time. A Dutch travel agency, for instance, sells holidays through ascending auctions, next to selling them at a posted price. ${ }^{14}$ In Canada, a charity offers its donors two auction formats from which they may choose: FPAs and ascending auctions. ${ }^{15}$ This suggests that the mechanism through which goods are sold has become the subject of versioning. Therefore, we extend our model to a monopoly setting, where we limit ourselves to two simultaneous auctions only. Consider a monopolist who sells $M=2$ units of a homogeneous good and decides to offer these in two simultaneous auctions. He can either choose to offer two FPAs, two SPAs, or a combination of an FPA and an SPA. The monopolist's objective is to choose the strategy profile with the highest expected revenue among these alternatives. Alternatively, the monopoly setting can be interpreted as representing the auction selection decisions of competing sellers when they collude.

\footnotetext{
14 Emesa Nederland BV offers an array of commodities and services in both online ascending auctions and at posted prices (see www.vakantieveilingen.nl and www.actievandedag.nl, respectively, both visited in June 2018).

15 Ducks Unlimited Canada organizes online charity auctions using both ascending and sealed bid auctions (see www.ducks.ca/how-to-help/auction, visited in June 2018).
} 
Proposition 3 Suppose that a monopolist sells his goods in two simultaneous auctions and chooses between FPAs and SPAs, and that bidders are risk averse $(r>0)$ and choose between auctions as described in Proposition 1. Then there exists a range of absolute rates of risk aversion around risk neutrality such that a monopolist prefers to offer both units in FPAs.

Proof Recall that by Lemma 1 , for $M=2$, it is the case that $q^{*}=0.5$ when $a_{1}=$ $a_{2}$. Additionally, recall that the traditional revenue ranking implies that $R_{I}\left(n_{I}, r\right)>$ $R_{I I}\left(n_{I I}\right)$ for $n_{I}=n_{I I}$ and $r>0$. It therefore follows immediately that the sum of expected revenues of (FPA, FPA) is greater than that of (SPA, SPA). Consequently, to prove Proposition 3, it suffices to show that the sum of expected revenues of (FPA, FPA) is greater than that of (FPA, SPA). The sum of expected revenues of offering both an FPA and an SPA is given by

$$
\begin{aligned}
& \sum_{n_{I}=0}^{N}\left(\begin{array}{c}
N \\
n_{I}
\end{array}\right)\left(q^{*}\right)^{n_{I}}\left(1-q^{*}\right)^{N-n_{I}} R_{I}\left(n_{I}, r\right) \\
& +\sum_{n_{I I}=0}^{N}\left(\begin{array}{c}
N \\
n_{I I}
\end{array}\right)\left(1-q^{*}\right)^{n_{I I}}\left(q^{*}\right)^{N-n_{I I}} R_{I I}\left(n_{I I}\right)
\end{aligned}
$$

The sum of expected revenues of two FPAs is given by

$$
0.5^{N} \sum_{n_{I}=0}^{N}\left(\begin{array}{l}
N \\
n_{I}
\end{array}\right) R_{I}\left(n_{I}, r\right)+0.5^{N} \sum_{n_{I}=0}^{N}\left(\begin{array}{l}
N \\
n_{I}
\end{array}\right) R_{I}\left(n_{I}, r\right)
$$

To prove by contradiction, assume that the sum of expected revenues of (FPA, SPA) is at least as large as that of (FPA, FPA). As the number of bidders ranges from 0 to $N$ in both FPAs and SPAs, from here on out we use one and the same $n$, instead of $n_{I}$ and $n_{I I}$.

$$
\begin{aligned}
& \sum_{n=0}^{N}\left(\begin{array}{l}
N \\
n
\end{array}\right)\left\{\left[\left(q^{*}\right)^{n}\left(1-q^{*}\right)^{N-n}-2 * 0.5^{N}\right] R_{I}(n, r)\right. \\
& \left.\quad+\left(1-q^{*}\right)^{n}\left(q^{*}\right)^{N-n} R_{I I}(n)\right\} \geq 0
\end{aligned}
$$

By the revenue equivalence theorem, $R_{I I}(n, 0)=R_{I I}(n)$, and by Lemma $3, q^{*}=0.5$ for $r=0$. At risk neutrality $(r=0)$, the sum of expected revenues of (FPA, FPA) must be equal to that of (FPA, SPA). Consequently, it suffices to show that at $r=0$ the derivative of (17) with respect to $r$ is nonnegative. Differentiating (17) with respect to $r$ produces the following equation. 


$$
\begin{aligned}
& \sum_{n=0}^{N}\left(\begin{array}{l}
N \\
n
\end{array}\right)\left\{\left[\left(q^{*}\right)^{n}\left(1-q^{*}\right)^{N-n}-2 * 0.5^{N}\right] \frac{\partial R_{I}(n, r)}{\partial r}\right. \\
& \quad+\left(q^{*}\right)^{n-1}\left(1-q^{*}\right)^{N-n-1}\left[n-q^{*} N\right] \frac{\mathrm{d} q^{*}}{\mathrm{~d} r} R_{I}(n, r) \\
& \left.\quad+\left(1-q^{*}\right)^{n-1}\left(q^{*}\right)^{N-n-1}\left[\left(1-q^{*}\right) N-n\right] \frac{\mathrm{d} q^{*}}{\mathrm{~d} r} R_{I I}(n)\right\} \geq 0
\end{aligned}
$$

We now evaluate this at $r=0$, which by Lemma 3 implies $q^{*}=0.5$.

$$
\begin{gathered}
\sum_{n=0}^{N}\left(\begin{array}{l}
N \\
n
\end{array}\right)\left\{-0.5^{N} \frac{\partial R_{I}(n, r)}{\partial r}+0.5^{N-2}[n-0.5 N] \frac{\mathrm{d} q^{*}}{\mathrm{~d} r} R_{I}(n, 0)\right. \\
\left.+0.5^{N-2}[0.5 N-n] \frac{\mathrm{d} q^{*}}{\mathrm{~d} r} R_{I I}(n)\right\} \geq 0
\end{gathered}
$$

where the last two terms cancel out as $R_{I}(n, 0)=R_{I I}(n)$ and where $\frac{\partial R_{I}(n, 0)}{\partial r}>0$. As a result, the equation above is strictly negative, contradicting our assumption. This concludes the proof of this proposition.

Proposition 3 states that, for some range around risk neutrality, a monopolist prefers to offer two FPAs to offering them in different auctions or in SPAs. This result is independent of whether bidders exhibit CARA, DARA or IARA. In case of CARA, however, we show that the result is more general.

Corollary 1 Suppose that a monopolist sells his goods in two simultaneous auctions and chooses between FPAs and SPAs, and that bidders are risk averse ( $r>0)$, exhibit CARA, and choose between auctions as described in Proposition 1. Then a monopolist prefers to offer both units in FPAs to offering them in an FPA and an SPA, which is preferred to offering them in SPAs.

Proof By Proposition 1, we know that with CARA bidders, for $M=2$, it is the case that $q^{*}=0.5$ for every $r$. As $R_{I}(n, r)>R_{I I}(n)$ for $r>0$, it follows immediately that the sum of expected revenues from (FPA, FPA) is greater than that of (FPA, SPA), which is in turn greater than that of (SPA, SPA).

Simulations for utility functions exhibiting CARA, DARA and IARA consistently show that a monopolist prefers to select only FPAs. We therefore conjecture that Corollary 1 holds as well for bidders exhibiting DARA or IARA. Our findings are consistent with traditional revenue ranking theorems, but seem less consistent with practice in online auctions. Whereas our results indicate that it is not profitable to use auction design as a means of versioning, this is exactly what happens on the Internet. Perhaps such versioning by monopolists can only be explained when bidders have nonstandard preferences. Future research might therefore consider behavioral assumptions such as reference-dependent preferences and competitiveness. Taking into account more sophisticated assumptions might better explain bidders' entry decisions and, hence, the form that auction versioning by monopolists takes. 


\section{References}

Arrow, K.J.: Essays in the Theory of Risk Bearing. Markham, Chicago (1971)

Aycinena, D., Bejarano, H., Rentschler, L.: Informed entry in auctions. Int. J. Game Theory 47(1), 175-205 (2018)

Bajari, P., Hortacsu, A.: The winner's curse, reserve prices, and endogenous entry: empirical insights from ebay auctions. RAND J. Econ. 34(2), 329-355 (2003)

Chen, K.Y., Plott, C.R.: Nonlinear behavior in sealed bid first price auctions. Games Econ. Behav. 25(1), 34-78 (1998)

Cox, J.C., Oaxaca, R.L.: Is bidding behavior consistent with bidding theory for private value auctions? Res. Exp. Econ. 6, 131-148 (1996)

Cox, J.C., Smith, V.L., Walker, J.M.: Experimental development of sealed-bid auction theory; calibrating controls for risk aversion. Am. Econ. Rev. 75(2), 160-165 (1985)

Cox, J.C., Smith, V.L., Walker, J.M.: Theory and individual behavior of first-price auctions. J. Risk Uncertain. 1(1), 61-99 (1988)

Damianov, D.S.: Seller competition by mechanism design. Econ. Theory 51(1), 105-137 (2012). https:// doi.org/10.1007/s00199-010-0597-z

Engelbrecht-Wiggans, R.: On optimal reservation prices in auctions. Manag. Sci. 33(6), 763-770 (1987)

Engelbrecht-Wiggans, R.: Optimal auctions revisited. Games Econ. Behav. 5(2), 227-239 (1993)

Engelbrecht-Wiggans, R., Katok, E.: Experiments on auction valuation and endogenous entry. In: Morgan, J. (ed.) Experimental and Behavioral Economics, Advances in Applied Microeconomics, chap 7, vol. 13, pp. 169-193. Emerald Group Publishing Limited, Bingley (2005)

Farouki, R.T.: The Bernstein polynomial basis: a centennial retrospective. Comput. Aided Geom. Des. 29(6), 379-419 (2012)

Goeree, J.K., Holt, C.A., Palfrey, T.R.: Risk averse behavior in generalized matching pennies games. Games Econ. Behav. 45(1), 97-113 (2003)

Harsanyi, J.C.: Games with randomly disturbed payoffs: a new rationale for mixed-strategy equilibrium points. Int. J. Game Theory 2(1), 1-23 (1973)

Holt, C., Laury, S.: Risk aversion and incentive effects. Am. Econ. Rev. 92(5), 1644-1655 (2002)

Hon-Snir, S.: Utility equivalence in auctions. Contrib. Theor. Econ. 5(1), 1-11 (2005)

Ivanova-Stenzel, R., Salmon, T.C.: Bidder preferences among auction institutions. Econ. Inquiry 42(2), 223-236 (2004a)

Ivanova-Stenzel, R., Salmon, T.C.: Entry fees and endogenous entry in electronic auctions. Electron. Markets 14(3), 170-177 (2004b)

Ivanova-Stenzel, R., Salmon, T.C.: Revenue equivalence revisited. Games Econ. Behav. 64(1), 171-192 (2008a)

Ivanova-Stenzel, R., Salmon, T.C.: Robustness of bidder preferences among auction institutions. Econ. Inquiry 46(3), 355-368 (2008b)

Ivanova-Stenzel, R., Salmon, T.C.: The high/low divide: self-selection by values in auction choice. Games Econ. Behav. 73(1), 200-214 (2011)

Kagel, J.H., Levin, D.: Independent private value auctions: bidder behaviour in first-, second- and third-price auctions with varying numbers of bidders. Econ. J. 103(419), 868-879 (1993)

Kagel JH, Levin D (2014) Auctions: a survey of experimental research. Working paper

Kagel, J.H., Harstad, R.M., Levin, D.: Information impact and allocation rules in auctions with affiliated private values: a laboratory study. Econometrica 55(6), 1275-1304 (1987)

Klemperer, P.: What really matters in auction design. J. Econ. Perspect. 16(1), 169-189 (2002)

Levin, D., Smith, J.L.: Equilibrium in auctions with entry. Am. Econ. Rev. 84(3), 585-599 (1994)

Levy, H.: Absolute and relative risk aversion: an experimental study. J. Risk Uncertain. 8(3), 289-307 (1994)

Maskin, E., Riley, J.: Optimal auctions with risk averse buyers. Econometrica 52(6), 1473-1518 (1984)

Matthews, S.: Selling to risk averse buyers with unobservable tastes. J. Econ. Theory 30(2), 370-400 (1983)

Matthews, S.: Comparing auctions for risk averse buyers: a buyer's point of view. Econometrica 55(3), 633-646 (1987)

McAfee, R.P.: Mechanism design by competing sellers. Econometrica 61(6), 1281-1312 (1993)

McAfee, R.P., McMillan, J.: Auctions with a stochastic number of bidders. J. Econ. Theory 43(1), 1-19 (1987a)

McAfee, R.P., McMillan, J.: Auctions with entry. Econ. Lett. 23(4), 343-347 (1987b) 
Menezes, F.M., Monteiro, P.K.: Auctions with endogenous participation. Rev. Econ. Des. 5(1), 71-89 (2000)

Milgrom, P.R.: Putting Auction Theory to Work. Cambridge University Press, New York (2004)

Milgrom, P.R., Weber, R.J.: A theory of auctions and competitive bidding. Econometrica 50(5), 1089-1122 (1982)

Monderer, D., Tennenholtz, M.: K-price auctions: revenue inequalities, utility equivalence, and competition in auction design. Econ. Theory 24(2), 255-270 (2004). https://doi.org/10.1007/s00199-003-0424-X

Myerson, R.: Optimal auction design. Math. Oper. Res. 6(1), 58-73 (1981)

Palfrey, T.R., Pevnitskaya, S.: Endogenous entry and self-selection in private value auctions: an experimental study. J. Econ. Behav. Organ. 66(3-4), 731-747 (2008)

Peters, M., Severinov, S.: Competition among sellers who offer auctions instead of prices. J. Econ. Theory 75(1), 141-179 (1997)

Pevnitskaya S (2004) Endogenous entry in first-price private value auctions: the self-selection effect. Working paper

Reiley, D.H.: Experimental evidence on the endogenous entry of bidders in Internet auctions. In: Rapoport, A., Zwick, R. (eds.) Economic and Managerial Perspectives, Experimental Business Research, vol. 2, pp. 103-121. Springer, Boston (2005)

Riley, J.G., Samuelson, W.F.: Optimal auctions. Am. Econ. Rev. 71(3), 381-392 (1981)

Smith, J.L., Levin, D.: Ranking auctions with risk averse bidders. J. Econ. Theory 68(2), 549-561 (1996)

Smith, J.L., Levin, D.: Entry coordination in auctions and social welfare: an experimental investigation. Int. J. Game Theory 30(3), 321-350 (2002)

Vickrey, W.: Counterspeculation, auctions and competitive sealed tenders. J. Bus. 59(4), 251-278 (1961)

Wakker, P.P.: Explaining the characteristics of the power (CRRA) utility family. Health Econ. 17(12), 1329-1344 (2008) 